\title{
Modeling and analysis of the acidizing process in carbonate rocks using a two-phase thermal-hydrologic-chemical coupled model
}

\author{
Piyang Liu ${ }^{\mathrm{a}, \mathrm{b}}$, Xia Yan ${ }^{\mathrm{a}}$, Jun $\mathrm{Yao}^{\mathrm{a}}$, Shuyu Sun ${ }^{\mathrm{b}, *}$ \\ ${ }^{a}$ School of Petroleum Engineering, China University of Petroleum (East China), QingDao, 266580, China \\ ${ }^{b}$ Computational Transport Phenomena Laboratory(CTPL), Division of Physical Sciences and Engineering \\ (PSE), King Abdullah University of Science and Technology (KAUST), Thuwal 23955-6900, Kingdom of \\ Saudi Arabia
}

\begin{abstract}
We present a two-phase thermal-hydrologic-chemical coupled model for simulating the dissolution process during the acidization of carbonate rocks. In particular, we develop a new model to describe the change in irreducible water saturation, residual oil saturation, and the maximum relative permeability of oil and water phases with dissolution proceeding. We also present a new method for the generation of the initial porosity field with controllable correlation length. In numerical calculation, the sequential iteration approach is adopted to solve the presented model, and splitting method is used to deal with the reaction relevant equations. The involved equations are discretized using the finite-volume method, where the convection term is discretized by the MINMOD scheme which can prevent overshoot/undershoot of the numerical solution. Additionally, sensitivity analysis of the dissolution process concerning rock properties, the exothermic heat of reaction, and twophase flow, is carried out. Based on the predicted results, several recommendations for the carbonate acidizing operation are given, and the potential extensions of the current work are summarized.
\end{abstract}

Keywords: Carbonate acidizing, Wormhole, Two-phase flow, T-H-C coupled, Reactive flow

\section{Introduction}

Acidizing is the most common methods developed to stimulate carbonate reservoirs. It is conducted by injecting acid into the formation at pressures that are lower than the fracturing

* Corresponding author

Email address: shuyu. sun@kaust.edu.sa (Shuyu Sun)

Preprint submitted to Chemical Engineering Science

April 6, 2019 
pressure. As the acid is injected into the formation, it penetrates part of pores and dissolves some minerals, and usually, if successful, creates wormholes. These resulting wormholes can bypass the drilling-caused or production-caused damaged zone near the wellbore, providing a low resistance path for hydrocarbon flow into the well [1]. Therefore, there is a practical motivation to seek sets of conditions and characteristics that lead to the development of wormholes.

The injection rate of acid is one of the earliest noticed factor affecting the formation of wormholes. By injecting the acid into carbonate cores at different rates in the laboratory $[2,3,4]$, it has been found that five types of dissolution patterns, named face dissolution, conical wormhole, wormhole, ramified wormhole, and uniform dissolution, can be formed depending on the injection rate. If the injection velocity is meager, all acid will be consumed before penetrating deeply into the medium. As a result, the entire face of the rock is dissolved leading to the formation of the face dissolution pattern. On the contrary, when acid is injected at a very high rate, it does not have sufficient time to react with the rock before being flushed out by the subsequent acid. In this case, the injected acid can reach almost all of the connected pores, which results in a uniform increase in porosity and consequently leading to the uniform dissolution pattern. Only at an appropriate injection rate, the acid dissolves the rock preferentially, leading to the development of the wormhole pattern. When the injection rate is between the rates of formation of wormholes and uniform dissolution patterns, ramified wormholes can be observed, while when the injection rate is between the wormhole and face dissolution patterns, conical wormholes are formed. By measuring the acid volume required to increase the effective permeability of the core by a specific factor, i.e., the breakthrough volume $P V_{B T}$, these experimental studies also found that the volume of acid needed is minimal when forming the wormhole dissolution pattern. Thus, the injection rate at which wormhole is formed is termed as the optimum injection rate, and the determination of it is critical to achieving successful stimulation of carbonate reservoir.

To explore the mechanism that governs the wormhole formation and seek for the optimum injection rate, a considerable amount of experiments have been conducted $[5,6,7,8$, 9, 10, 11, 12, 13, 14, 15]. By treating the carbonate cores with different acid $[2,4,9,16]$, it is found that the formation of wormholes is determined by the combined effect of diffusivity and reactivity. By injecting the acid into rocks with different mineral component and heterogeneity $[16,17]$, it is found that the formed dissolution pattern varies with the mineral composition and rock heterogeneity even though the injection rate is the same. By injecting the acid linearly and radially into the rock [12], it is found that the optimum 
injection rate observed in radial flow experiments is higher than that in linear flow case. The breakthrough volume $P V_{B T}$ obtained under the optimum conditions is also lower in case of radial flow than in case of linear flow. By performing the acid flooding experiment at different temperatures $[3,12,16,18]$, it is found that with the increase of temperature, the value of optimum injection rate increases, but the breakthrough volume may increases or decreases, depending on the type of rock-acid system. By injecting the acid into a core that is saturated with a non-aqueous phase $[19,20,21]$, it is found that the presence of a non-aqueous phase will lead to the development of narrower and lower branched wormholes. Although these experimental results provide a fundamental understanding of the dissolution process, the optimum injection rate obtained under laboratory conditions might not be translated directly into recommendations on performing a real acidizing treatment. This is partly due to the fact that the scale of the cores used in the experiment is much smaller than the field scale and hence dissolution structures observed in the acid flooding experiment may not be representative of those formed in the field. On the other hand, it is very hard to replicate the actual reservoir conditions, including pressure, temperature, and stress, in the laboratory. Alternately, numerical simulation of the dissolution process provides an efficient tool for seeking the optimum operating conditions of the acidizing treatment.

Numerous mathematical models have also been presented in the literature to simulate the dissolution dynamic and account for the wormhole formation. The most widely used one is the two-scale continuum model (TSC) developed by Panga et al. [22] as it is advantageous at predicting the dissolution patterns observed in experiments and its accurate estimation of $P V_{B T}$. For this reason, the TSC model is continuously extended to take into account the factors that affect the wormhole propagation [23, 24, 25, 26, 27, 28, 29, 30, 31, 32, $33,34,35,36,37,38,39,40,41,42,43,44,45,46]$. For example, Kalia and Balakotaiah [23], and Cohen et al. [24] extended the TSC model to the 2-D radial case by using the $r-\theta$ coordinates. They also compared the dissolution patterns and $P V_{B T}$ obtained under linear flow conditions with that obtained under radial flow conditions. Liu et al. [25, 26] introduced new methods for generating the initial porosity field used in the simulation to make the prediction of the TSC model closer to experimental observations. Ratnakar et al. [27, 28], and Maheshwari et al. [29] modified the momentum equation and included a rheological equation in the TSC model to make it capable of simulating the acidization with diverting acids, which are non-Newtonian in nature. Combining the discrete fracture model with the TSC model, Liu et al. [30, 31] obtained a two-scale discrete fracture continuum model which can be used to evaluate the influence of fractures on wormhole formation. Kalia 
$[32,33]$, and $\mathrm{Li}$ et al. $[34,35]$ coupled the heat transport to the TSC model to investigate the effect of temperature on the dissolution process. Wei et al. [36] , Mahmoodi et al. [37], and Babaei et al. [38] used a two-phase flow field to study the influence of the presence of a nonaqueous phase on wormhole structure. In summary, reactive dissolution of carbonate rocks has been extensively studied both experimentally and theoretically to predict conditions under which wormholes are created. Several known factors affecting dissolution pattern formation, such as acid injection rate, heterogeneity, system geometry, reaction kinetics, temperature, multi-phase flow, et al., have been the subject of investigation. However, there is no comprehensive model in the literature that couples these known mechanisms together to investigate the influence of above-mentioned factors on the dissolution process under actual formation conditions.

The main aim of this paper is to develop a comprehensive model that can take into account the effect of reaction kinetics, rock heterogeneity, system geometry, reaction temperature, and the presence of another phase on wormhole formation during acidization. This is accomplished by extending the commonly used TSC model developed by Panga et al. [22] to a two-phase thermal-hydrologic-chemical coupled model. Using this model, the dissolution process under actual reservoir conditions can be easily investigated. The coupled thermal-hydrological-chemical processes are involved in various geological, scientific, and engineering areas. In addition to the acidization of carbonate reservoir, some prominent examples include melt migration [47, 48], environmental contaminant transport [49, 50], geologic sequestration of carbondioxide [51, 52, 53], disposal of nuclear wastes[54] and so on. In the past three decades, researches on the development of reactive transport model coupled with heat transfer and/or mechanistic have exploded [55]. Various effective numerical algorithms have also been developed for solving different specific problems [56, 57, 58, 59, 60]. These models are capable of handling a complete suite of geochemical reaction processes. Because the geochemical processes are usually slow and reversible, the effects of chemical reactions on rock properties and fluid flow may take decades or even hundreds of years to manifest. However, different from the geochemical reaction, the reaction taking place during carbonate acidization is fast in comparison with transport phenomena. As a result, complete dissolution may occur in the rock under certain conditions, which brings challenges to modeling and numerical simulation. It is also the aim of this paper to develop a model to describe the variation of relative permeability with dissolution in the presence of multi-phase flow.

This paper is organized as follows. In Section 2, the mathematical model is presented 
detailedly, to describe the involved chemical system, fluid flow, mass and energy transport, rock dissolution, and other phenomenon induced by the reaction. In Section 3, the dimensionless model is derived by defining a series of dimensionless parameters. In Section 4, the numerical method used for solving the presented model is given. The effect of using the upwind scheme on simulation accuracy is also analyzed in this section. In Section 5 we compare the calculation results of a degenerate model with an analytical solution and available experimental observations to verify our work. In Section 6, numerical experiments are performed to highlight the effect of rock properties, the exothermic heat of reaction, and two-phase flow on the dissolution process. Finally, the paper is summarized by conclusions in Section 7.

\section{Mathematical model}

The following assumptions are made for deriving the two-phase thermal-hydrologicchemical coupled model for simulating the acidizing process in carbonate rocks: (1) the fluid is incompressible, i.e., the change in pressure and temperature does not affect the fluid density; (2) the compressibility of the rock is neglected because the porosity change caused by rock compressibility is much less than that caused by the chemical reaction during the acidizing procedure; (3) the water phase is regarded as the wet phase, and the oil phase is regarded as the non-wet phase; (4) fluid flow in carbonate rocks obeys Darcy's law, and the gravity and capillary pressure are neglected because their values are small; (5) the injected acid is only dissolved in the water phase; (6) the thermal equilibrium between the fluid and rock can be achieved instantaneously.

\subsection{Chemical system}

Because the hydrochloric acid $(\mathrm{HCl})$ can be completely ionized into hydrogen ions $\left(\mathrm{H}^{+}\right)$ and chloride ions $\left(\mathrm{Cl}^{-}\right)$in aqueous solution, the stoichiometry of reaction between the hydrochloric acid and calcite, which occurs at the fluid-solid surface, can be written as

$$
2 \mathrm{H}^{+}+\mathrm{CaCO}_{3}(s) \stackrel{r_{s}}{\rightarrow} \mathrm{Ca}^{2+}+\mathrm{H}_{2} \mathrm{O}+\mathrm{CO}_{2}(g)
$$

where $r_{s}$ denotes the reaction rate of the reaction Eq. (1), which is defined based on the interfacial surface area, i.e., the change in the concentration of the reactants or products per unit time per unit interfacial surface area. In the definition of $r_{s}$, the change rate in concentration of a substance is divided by its stoichiometric coefficient so that the defined rate is independent of which substance is chosen for measurement. According to Eq. (1), the 
rate of disappearance of $\mathrm{H}^{+}$is $2 r_{s}$. It is acceptable to approximate the $\mathrm{HCl}-\mathrm{CaCO}_{3}$ reaction as an irreversible first order reaction. Under this hypothesis, the rate law for reaction Eq. (1) is given as

$$
r_{s}=k_{s} c_{s}
$$

where $c_{s}$ denotes the concentration of the $\mathrm{H}^{+}$at the fluid-solid surface. $k_{s}$ is the surface reaction rate constant, which is temperature dependent and can be calculated by the Arrhenius equation as

$$
k_{s}=A \cdot \exp \left(-\frac{E_{a}}{R T}\right)
$$

where $A$ is named as the pre-exponential factor, $E_{a}$ is the activation energy, and the value of both them can be determined experimentally[32]. $R$ is the universal gas constant, $T$ is the temperature (in Kelvins), at which the reaction takes place. Through Eq. (3), If the rate constant at a temperature of $T^{0}$ is known as $k_{s}^{0}$, the rate constant at any temperature can be calculated by

$$
k_{s}=k_{s}^{0} \exp \left(-\frac{E_{a}}{R}\left(\frac{1}{T}-\frac{1}{T^{0}}\right)\right)
$$

\subsection{Fluid flow}

The formation was initially saturated by oil before the acid is injected. Therefore, during the acidizing procedure, two-phase flow exists in the formation. For each phase, the flow is governed by the mass balance law and Darcy's law as [61]

$$
\frac{\partial\left(\phi S_{\alpha}\right)}{\partial t}+\nabla \cdot \mathbf{u}_{\alpha}=0
$$

$$
\mathbf{u}_{\alpha}=-\frac{K_{\alpha} \mathbf{K}}{\mu_{\alpha}} \nabla P_{\alpha}
$$

where the subscript $\alpha \in\{w, o\}$, in which $w$ and $o$ denote the water (acid) and oil phases, respectively. $t$ is the time, $\phi$ is the formation porosity, $S_{\alpha}$ is the saturation of phase $\alpha, \mathbf{u}_{\alpha}$ is the velocity of phase $\alpha, \mathbf{K}$ is the absolute permeability of the formation, $K_{\alpha}$ is the relative permeability of phase $\alpha . \mu_{\alpha}$ is the viscosity of phase $\alpha, P_{\alpha}$ is the pressure for phase $\alpha$, and when neglecting the capillary pressure, we have

$$
P_{o}=P_{w}=P
$$

The viscosity of both the oil and injected acid is temperature dependent. The viscosity of injected acid at a given temperature (in Kelvin) can be approximated by [62]

$$
\mu_{w}(T)=2.4 \times 10^{-2} \times 10^{247.8 /(T-140)}
$$


where $\mu_{w}$ has the units of $\mathrm{mPa} \cdot \mathrm{s}$.

For the oil phase, the correlation between viscosity and temperature is not unique, but depends on many factors such as the oil composition and the dissolved gas. Here we adopt an exponential formula to describe the change of crude oil viscosity with temperature as [28]:

$$
\mu_{o}(T)=\mu_{o}^{0} \exp \left(-\alpha_{\mu} \frac{T-T^{0}}{T^{0}}\right), \quad 0<\alpha_{\mu} \ll 1
$$

where $\mu_{o}^{0}$ is the viscosity of oil at temperature $T^{0}$. It should be point out that, in practical application, the temperature dependence of fluid viscosity should be determined or corrected based on the experimental data.

A number of models have been developed to describe the relative permeabilities of oil and water phase. In these models, relative permeabilities are commonly assumed as a function of phase saturation. The most widely used model named modified Brooks-Corey relations are adopted in this work[63], and the oil and water relative permeabilities are expressed as:

$$
K_{r o}=K_{r o}^{\max }\left(\frac{1-S_{w}-S_{o r}}{1-S_{w i}-S_{o r}}\right)^{n_{o}}
$$

$$
K_{r w}=K_{r w}^{\max }\left(\frac{S_{w}-S_{w i}}{1-S_{w i}-S_{o r}}\right)^{n_{w}}
$$

where $K_{o}^{\max }$ is the oil relative permeability at irreducible water saturation $S_{w i}, K_{w}^{\max }$ is the water relative permeability at maximum water saturation, i.e., $S_{w}=1-S_{o r}$, and $S_{o r}$ is the residual oil saturation. $n_{o}$ and $n_{w}$ are Corey oil exponent and Corey water exponent, respectively. Typical ranges of Corey exponents are from 1 to 6 . In the following simulations, we take the values of $n_{o}=2.4$ and $n_{w}=2.4$. The irreducible or residual saturation of a phase is the amount of that phase that is trapped in the pores. Many properties such as pore structure, permeability, and wettability of the rock affect the value of irreducible or residual saturation. Various techniques are available to determine the residual oil saturation or irreducible water saturation, such as core analysis methods, well logging methods. In usual reservoir simulations, these estimated residual saturations are constant. However, in the reactive transport process, the pore structure is continuously altered due to rock dissolution. It is obvious that no fluid will be trapped in the pores if the rock is completely dissolved. Therefore, the alteration of $S_{w i}$ and $S_{o r}$ with the rock dissolution should be considered when simulating the reactive transport process in oil-water phase system. This assumption is also applicable to $K_{o}^{\max }$ and $K_{w}^{\max }$ because they represent the relative permeability at $S_{w i}$ and $1-S_{o r}$. In other words, the relative permeability curve changes dynamically as 
93

the dissolution proceeding. However, there are no accurate correlations to describe these changes. Mahmoodi et al. [37] used a piecewise linear function to describe this phenomenon. Here, we propose a more general model to express the change of relative permeability curve with porosity variation as

$$
S_{w i}=S_{w i, 0} \frac{\tanh \left(a_{w}(1-\phi)\right)}{\tanh a_{w}}
$$

$$
S_{o r}=S_{o r, 0} \frac{\tanh \left(a_{o}(1-\phi)\right)}{\tanh a_{o}}
$$

$$
K_{r w}^{\max }=1+\left(1-K_{r w, 0}^{\max }\right) \frac{\tanh \left(a_{k w}(\phi-1)\right)}{\tanh a_{k w}}
$$

$$
K_{r o}^{\max }=1+\left(1-K_{r o, 0}^{\max }\right) \frac{\tanh \left(a_{k o}(\phi-1)\right)}{\tanh a_{k o}}
$$

where $a_{w}, a_{o}, a_{k w}$, and $a_{k o}$ are constants that control the curvature of the curve of the dependent variable as a function of porosity. For simplicity, these constants take the same value in the current work and are denoted as $a$. To show the influence of $a$ clearly, the profiles of $S_{w i}$ and $K_{r w}^{\max }$ as a function of porosity are displayed in Fig. 1 for $a$ taking different values. It can be clearly seen that variations in $S_{w i}$ and $K_{r w}^{m a x}$ with increasing porosity change from almost a linear profile when $a$ approaches 0 to almost a step profile at high values of $a$. Fig. 2 shows the relative permeability curves for different values of porosity when $a=10$, which is the adopted value in this work. The effect of $a$ value on dissolution dynamic will be discussed later.

A constraint for saturation is needed to close the two-phase flow equations and is written as

$$
S_{w}+S_{o}=1
$$

\subsection{Reactive transport}

By applying the mass balance law to the specific chemical species, the reactive transport of $\mathrm{H}^{+}$can be expressed via the cup-mixing concentration as

$$
\frac{\partial\left(\phi S_{w} c_{f}\right)}{\partial t}+\nabla \cdot\left(\mathbf{u}_{w} c_{f}\right)=\nabla \cdot\left(\phi S_{w} \mathbf{D}_{e} \nabla c_{f}\right)-R_{a}
$$

with the cup-mixing concentration $c_{f}$ defined by the velocity weighted average as[64]

$$
c_{f}=\frac{\int_{V} \mathbf{u}(x, y, z) c_{H^{+}}(x, y, z) d V}{\int_{V} \mathbf{u}(x, y, z) d V}
$$




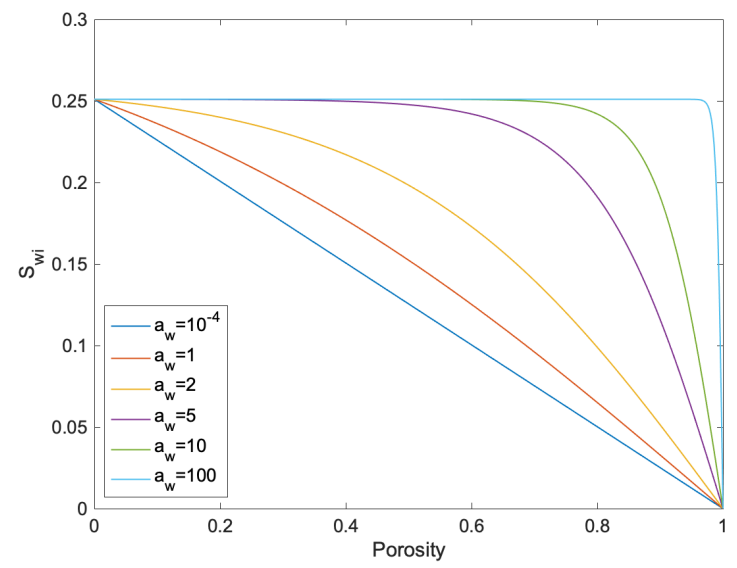

(a)

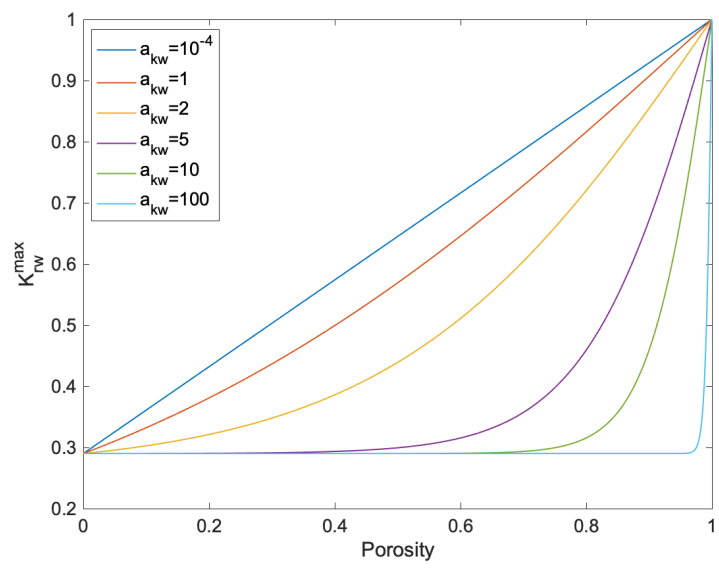

(b)

Figure 1: Effect of $a$ value on (a) irreducible water saturation and (b) maximum relative permeability of water

where $R_{a}$ represents the rate of disappearance of the species $\mathrm{H}^{+}$, which can be related to the surface reaction rate $r_{s}$ by the interfacial area available for reaction per unit volume of the medium $a_{v}$, and is found to be

$$
R_{a}=2 r_{s} a_{v}
$$

where the constant 2 appeared on the right-hand side is due to the stoichiometric coefficient of $\mathrm{H}^{+}$in Eq. (1). As the reaction proceeds, $\mathrm{H}^{+}$is consumed at the interface, and has to be compensated by transport from the bulk fluid, the rate of which is given by [41]

$$
N_{c}=k_{c}\left(c_{f}-c_{s}\right)
$$

where $N_{c}$ is the molar flux of the species, $k_{c}$ is the mass transfer coefficient defined based on the concentration driving force.

For steady state, the species transported to the fluid-solid interface is equal to that consumed on the surface due to the reaction, i.e.,

$$
k_{c}\left(c_{f}-c_{s}\right)=2 r_{s}=2 k_{s} c_{s}
$$

which can be rearranged into

$$
c_{s}=\frac{k_{c}}{k_{c}+2 k_{s}} c_{f}
$$

Substituting Eq. (22) into Eq. (2) yields

$$
r_{s}=\left(\frac{1}{k_{c}}+\frac{1}{2 k_{s}}\right)^{-1} c_{f}=k_{e} c_{f}
$$




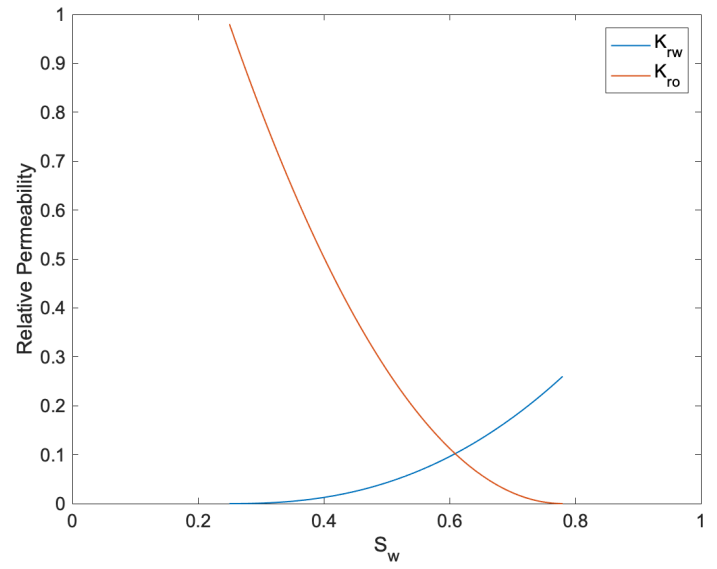

(a) $\phi=0.3$

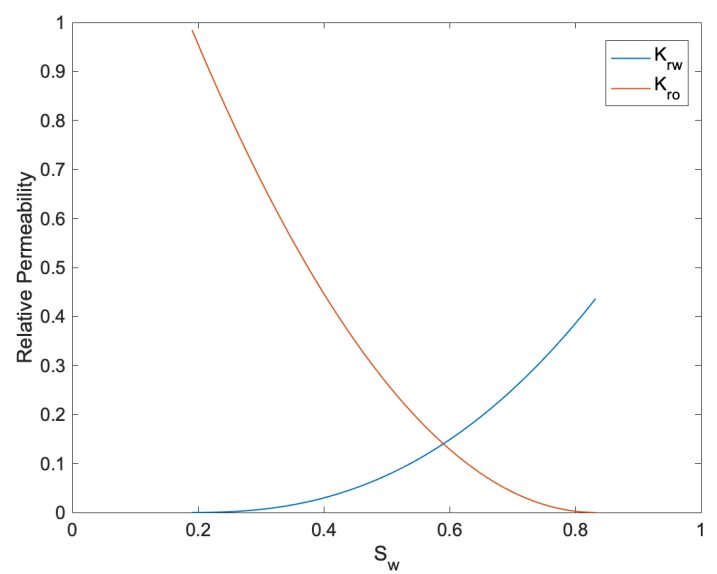

(c) $\phi=0.9$

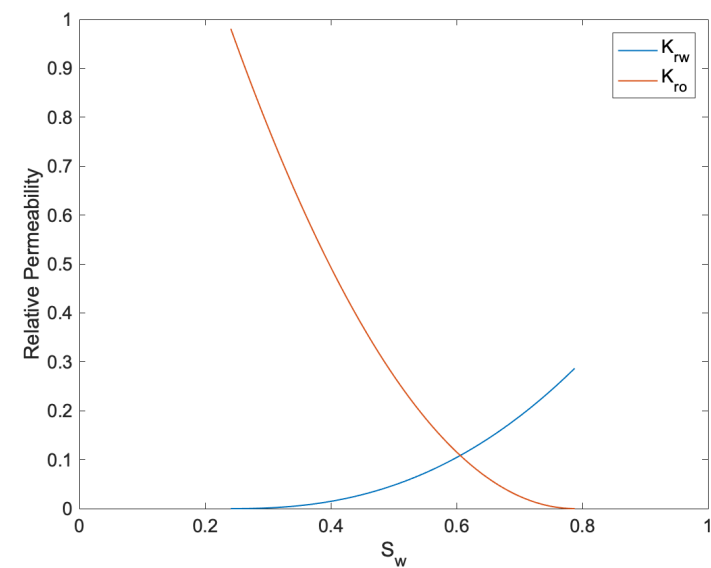

(b) $\phi=0.8$

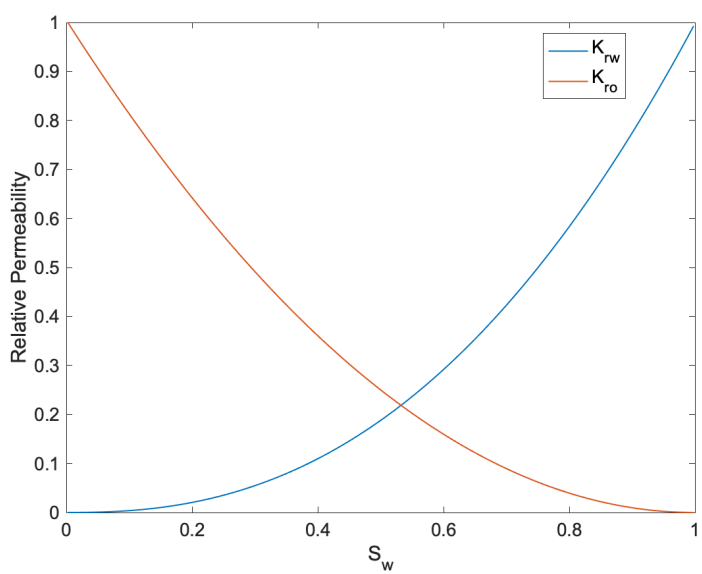

(d) $\phi=0.999$

Figure 2: The relative permeability curve for various porosity values when $a=10$

here an effective rate constant $k_{e}$ has been defined as

$$
k_{e}=\left(\frac{1}{k_{c}}+\frac{1}{2 k_{s}}\right)^{-1}
$$

Eq. (24) indicates that the value of the overall rate constant $k_{e}$ may vary depending on the relative magnitudes of $k_{c}$ and $k_{s}$. When the rate of reaction is much greater than the rate of mass transfer, i.e., $k_{s} \gg k_{c}$, Eq. (24) gives $k_{e} \approx k_{c}$, and Eq. (22) gives $c_{s} \approx 0$. The overall reaction rate is dominated by the mass transfer rate, so this is named as the diffusion-controlling reaction. On the contrary, if the surface reaction is much slower than the mass transfer, i.e., $k_{s} \ll k_{c}$, Eq.(24) gives $k_{e} \approx 2 k_{s}$, and Eq. (22) gives $c_{s} \approx c_{f}$. The surface reaction rate controls the overall reaction rate, and this is named as the reaction 
controlling reaction. For the isothermal case, because the reaction rate $k_{s}$ is a constant for a specified acid, the overall reaction rate $k_{e}$ only determined by the mass transfer coefficient $k_{c}$ which varies with the local fluid velocity, fluid properties, and the pore geometry. However, if the non-isothermal condition is considered, the dependence of the overall reaction rate on the surface reaction rate and mass transfer rate will become more complicated because both are related to temperature. Therefore, it is necessary to accurately calculate the change in $k_{s}$ and $k_{c}$ with temperature.

The value of $k_{c}$ depends on the fluid velocity, physical properties of the fluid, and the pore geometry, all of which may change as reaction proceeding. Dimensional analysis is commonly used to express this dependence and evaluate the mass transfer coefficient. The dimensionless form of the mass transfer coefficient is named as the Sherwood number Sh, and is written as [65]

$$
S h=\frac{2 k_{c} r_{p}}{D_{m}}=S h_{\infty}+0.7 \operatorname{Re}_{p}^{1 / 2} S c^{1 / 3}
$$

where $r_{p}$ is the pore radius; $S h_{\infty}$ is the asymptotic Sherwood number which accounts for the contribution from molecular diffusion, and has typical values of 2.98, 2.5, and 3.66 for pores with a square, triangular and circular cross-sections, respectively. The value of $S h_{\infty}$ is taken to be 3 in this work. $\operatorname{Re}_{p}$ is the pore Reynolds number defined as $\operatorname{Re}_{p}=2 u r_{p} / \nu$, with $\nu$ is the kinematic viscosity; $S c$ named Schmidt number and is defined as $S c=\nu / D_{m}$, and $D_{m}$ is the molecular diffusivity. In order to estimate the range of $R e$ and $S h$, the experimental data reported in [4] and presented by Kalia et. al [23] are used, i.e., $\nu=0.01 \mathrm{~cm}^{2} / \mathrm{s}$, $D_{m}=3.6 \times 10^{-5} \mathrm{~cm}^{2} / \mathrm{s}$. When the injection rate increases from $5 \times 10^{-4} \mathrm{~cm} / \mathrm{s}$ to $1 \mathrm{~cm} / \mathrm{s}$, which are typical values used in the simulation, the range of initial Reynolds number for pores of size in the range $1 \sim 200 \mu \mathrm{m}$ are $1 \times 10^{-5} \sim 4$, and the corresponding range of $S h$ are $3.015 \sim 12.14$. Detailed parameter values used in this work are list in Section 6.

A few of research has been done on the influence of temperature on the diffusion coefficient. It has been found that within a narrow temperature range - from $283 \mathrm{~K}$ to $293 \mathrm{~K}$ - the diffusion coefficient can be assumed to vary linearly with temperature as

$$
D_{m}=D_{m}^{0}\left[1+20 \eta_{T^{0}}^{1 / 2} \rho^{-1 / 3}\left(T-T^{0}\right)\right]
$$

where $D_{m}^{0}$ is the diffusion coefficient at $T^{0}=298 \mathrm{~K}, \eta_{T_{0}}$ is the solvent viscosity at $T^{0}=298 \mathrm{~K}$, $\mathrm{Ns} / \mathrm{m} ; \rho$ is the solvent density, $\mathrm{g} / \mathrm{cm}^{3}$.

Theoretically, the diffusion coefficient obeys the exponential Arrhenius relation and is given by:

$$
D_{m}=D_{m}^{0} \exp \left(-\frac{E_{D}}{R}\left(\frac{1}{T}-\frac{1}{T^{0}}\right)\right)
$$


The experimental data of Wilke and Chang [66] give available evidence that the activation energy of diffusion $E_{D}$ varies from 12.6 to $28.1 \mathrm{~kJ} / \mathrm{mol}$.

Substituting Eqs. (23) and (19) into Eq. (17), the reactive transport equation is transformed to

$$
\frac{\partial\left(\phi S_{w} c_{f}\right)}{\partial t}+\nabla \cdot\left(\mathbf{u}_{w} c_{f}\right)=\nabla \cdot\left(\phi S_{w} \mathbf{D}_{e} \nabla c_{f}\right)-\frac{2 a_{v} k_{s} k_{c}}{2 k_{s}+k_{c}} c_{f}
$$

In Eqs. (17) and (28), $\mathbf{D}_{e}$ is the effective dispersion tensor for species $\mathrm{H}^{+}$in the waterphase, and its longitudinal and transverse components are given, respectively by [36]

$$
D_{e L}=\alpha_{o s} D_{m}+\frac{2 \lambda_{L}\left|\mathbf{u}_{w}\right| r_{p}}{\phi}
$$

(1)

$$
D_{e T}=\alpha_{o s} D_{m}+\frac{2 \lambda_{T}\left|\mathbf{u}_{w}\right| r_{p}}{\phi}
$$

where $\left|\mathbf{u}_{w}\right|$ is the magnitude of the water-phase velocity. $\alpha_{o s}, \lambda_{L}$, and $\lambda_{T}$ are pore structure dependent constants, and they have typical values of $0.5,0.5$, and 0.1 for a packed-bed of spheres, respectively.

\subsection{Energy transport}

The temperature of the injected acid is usually different from that of the formation, so there is an energy exchange between the injected acid, initial saturated oil, and rock solid. Additionally, the reaction of $\mathrm{HCl}$ with calcite will release heat. As a result, the temperature inside the formation varies with space and time. Because the temperature affects chemical reaction rate and acid transport, the non-isothermal flow conditions should be considered when modeling the acidizing process. The heat transport equation for the three-phase system considered here - the two fluids and the solid matrix - is given by:

$$
\begin{aligned}
& \frac{\partial}{\partial t}\left(\sum_{\alpha=w, o} \phi S_{\alpha} \rho_{\alpha} C_{\alpha}^{p} T+(1-\phi) \rho_{s} C_{s}^{p} T\right)+\sum_{\alpha=w, o} \rho_{\alpha} C_{\alpha}^{p} \nabla \cdot\left(\mathbf{u}_{\alpha} T\right) \\
& =\left(\sum_{\alpha=w, o} \phi S_{\alpha} \Gamma_{\alpha}+(1-\phi) \Gamma_{s}\right) \nabla \cdot(\nabla T)+2\left(-\Delta H_{r}(T)\right) a_{v} k_{e} c_{f}
\end{aligned}
$$

where $\rho_{\alpha}$ is the density of the phase $\alpha, \rho_{s}$ is the density of the solid, $C_{\alpha}^{p}$ is the specific heat capacity of the phase $\alpha, C_{s}^{p}$ is the specific heat capacity of the solid, $T$ is the temperature, $\Gamma_{\alpha}$ is the thermal conductivity of the phase $\alpha, \Gamma_{s}$ is the thermal conductivity of the solid, $\Delta H_{r}(T)$ is the reaction exothermic heat at temperature $T$ defined as the heat generated 
per mole of $\mathrm{H}^{+}$consumed. $\Delta H_{r}(T)$ can be calculated by the difference in enthalpy between resultants and reactants. Mathematically, we have

$$
\Delta H_{r}(T)=\sum\left|\Delta H_{\text {resultants }}(T)\right|-\sum\left|\Delta H_{\text {reactants }}(T)\right|
$$

Because the enthalpy of any substance changes with temperature variation, $\Delta H_{r}$ is a function of temperature as presented in Eq. (32). The value of the enthalpy at different temperature can be evaluated through the Kirchhoff's law, by which Li [35] derived an expression to calculate the exothermic heat of reaction Eq. (1) at temperature $T$ as

$$
\Delta H_{r}(T)=-6846+8.038 T-3.22 \times 10^{-3} T^{2}-870.3 T^{-1}
$$

where the units of $\Delta H_{r}$ and $T$ are $\mathrm{J} / \mathrm{mole}^{+}$and $\mathrm{K}$, respectively.

\subsection{Rock dissolution}

The local reaction leads to alterations of the porosity, and the change of porosity with time is given by [30]

$$
\frac{\partial \phi}{\partial t}=\frac{R_{a} \alpha_{c}}{\rho_{s}}=\frac{2 a_{v} \alpha_{c} k_{s} k_{c}}{\rho_{s}\left(2 k_{s}+k_{c}\right)} c_{f}
$$

where $\alpha_{c}$ is the acid dissolving power defined as grams of calcite dissolved per mole of acid consumed.

\subsection{Reaction induced property changes}

The local porosity increase caused by calcite dissolution will change the rock properties such as permeability, pore radius, and specific surface area. It is computationally prohibitive to calculate the permeability evolution by solving equations at the pore scale. Moreover, as has been identified by previous investigators [67, 25], the permeability-porosity relations used in the acidizing simulation have a significant effect on wormhole propagate. Numerous works have been done on developing a proper permeability-porosity relation. Liu [25] summarized some commonly used formulas in the literature, and recommend to use a fractal theory based formulation to describe the evolution of permeability with porosity, which is adopted in this work.

By defining the fractal dimension for pore spaces and tortuosity, $D_{f}$ and $D_{T}$, respectively, as

$$
D_{f}=d_{E}-\frac{\ln \phi}{\ln \frac{\lambda_{\min }}{\lambda_{\max }}}
$$




$$
D_{T}=1+\frac{\ln \left(1-\frac{\phi}{2}+\frac{\sqrt{1-\phi}}{4}+\frac{(\phi+1+\sqrt{1-\phi}) \cdot \sqrt{9-5 \phi-8 \sqrt{1-\phi}}}{8 \phi}\right)}{\ln \left(\frac{D_{f}-1}{\sqrt{D_{f}}} \frac{\lambda_{\max }}{\lambda_{\min }} \sqrt{\frac{1-\phi}{\phi} \frac{\pi}{8-4 D_{f}}}\right)}
$$

320

the permeability can be related to the porosity as

$$
\frac{K}{K_{0}}=\frac{\left(\pi D_{f}\right)^{\frac{1-D_{T}}{2}}\left(8-4 D_{f}\right)^{\frac{1+D_{T}}{2}}\left(3+D_{T 0}-D_{f 0}\right)}{\left(\pi D_{f 0}\right)^{\frac{1-D_{T 0}}{2}}\left(8-4 D_{f 0}\right)^{\frac{1+D_{T 0}}{2}}\left(3+D_{T}-D_{f}\right)}\left(\frac{\phi}{1-\phi}\right)^{\frac{3+D_{T}}{2}}\left(\frac{\phi_{0}}{1-\phi_{0}}\right)^{-\frac{3+D_{T 0}}{2}}
$$

where the subscript 0 denotes the initial value of the corresponding parameter, $d_{E}$ is the Euclidean dimension. $\lambda_{\min }$ and $\lambda_{\max }$ are the smallest and the largest pore diameter, respectively, and $\lambda_{\min } / \lambda_{\max }$ have the typical value of 0.01 as analyzed in [25].

As shown in Eq. (19), the rate of species consumption requires the knowledge of the specific surface area $a_{v}$, which is calculated by [30]

$$
\frac{a_{v}}{a_{0}}=\frac{\phi r_{0}}{\phi_{0} r_{p}}
$$

with

$$
\frac{r_{p}}{r_{0}}=\sqrt{\frac{K \phi_{0}}{K_{0} \phi}}
$$

\subsection{Initial and boundary conditions}

Considering a polygonal and bounded domain $\Omega \subset \mathbb{R}^{d}(d=2,3)$, the following boundary conditions are used to represent that used in the acidizing experiments, where the acid is injected into the rock at a constant rate from the inlet, and the pressure at the outlet is fixed. For fluid flow,

$$
u_{0}=-\frac{K_{w} K}{\mu_{w}} \nabla P \cdot \mathbf{n}, \quad \text { on } \partial \Omega_{\text {in }}
$$

$$
P=P_{e}, \quad \text { on } \partial \Omega_{\text {out }}
$$

For solute transport,

$$
\left(\mathbf{u}_{w} c_{f}-\phi S_{w} \mathbf{D}_{e} \nabla c_{f}\right) \cdot \mathbf{n}=u_{0} c_{f 0}, \quad \text { on } \partial \Omega_{\text {in }}
$$

$$
\nabla c_{f} \cdot \mathbf{n}=0, \quad \text { on } \partial \Omega_{\text {out }}
$$


For energy transport,

$$
T=T_{f}, \quad \text { on } \partial \Omega_{\text {in }}
$$

$$
\nabla T \cdot \mathbf{n}=0, \quad \text { on } \partial \Omega_{\text {out }}
$$

where $u_{0}$ is the constant injection rate; $c_{f 0}$ and $T_{0}$ are the concentration and temperature of the injected acid, respectively; $P_{e}$ is the fixed pressure at the outlet boundary; $\mathbf{n}$ is the outward pointing unit vector normal to the specified boundary. For the transverse boundaries, the no-flux conditions are imposed to restrict the fluid, solute, and heat in the domain, and are expressed as

$$
\nabla P \cdot \mathbf{n}=0, \nabla c_{f} \cdot \mathbf{n}=0, \nabla T \cdot \mathbf{n}=0, \quad \text { on } \partial \Omega_{\operatorname{tran}}
$$

The initial conditions of acid concentration, rock temperature, and water saturation are given by

$$
c_{f}=0, T=T_{s}, S_{w 0}=S_{w i, 0}, \quad \text { at } t=0
$$

where $T_{s}$ is the initial temperature of the rock, which may varies with space.

If acid is injected into a homogeneous rock, only face dissolution pattern can be formed no matter how the injection rate varies. This is because that the dissolution front propagates stably in a uniform system. Therefore, in order to simulate all types of etched patterns observed in the acidizing experiments, the heterogeneity needs to be introduced into at least one of the porosity field, permeability field, and injection scheme when performing numerical simulations. In previous studies $[22,23,65,67,28]$, the most commonly used method to introduce the heterogeneity is perturbing the initial mean porosity with a random perturbation to generate a heterogeneous porosity field. Then the permeability field is related to the porosity value through the permeability-porosity correlations, for example, the commonly used Carman-Kozeny equation as:

$$
\frac{K}{K_{0}}=\frac{\phi^{3}}{\phi_{0}^{3}}\left(\frac{1-\phi_{0}}{1-\phi}\right)^{2}
$$

Up to now, many efforts $[22,26,44]$ have been given to generate a proper initial porosity field. For example, Panga et al. [22] added a uniformly distributed random fluctuation in the interval $[-\Delta \phi, \Delta \phi]$ to a uniform porosity field with the value of $\phi_{0}$ to get the heterogeneous porosity field when they performed numerical simulations. Using the heterogeneous porosity field as an initial condition, they simulated five types of dissolution patterns, which are the same with that observed in experiments. Liu et al. [26] improved Panga's method of 
generating the initial porosity field by adding a normally distributed random disturbance to the average porosity value. Because the porosity in real rocks is normally distributed rather than uniformly, more reasonable results on breakthrough volume are obtained when comparing with Panga's work. However, based on the characteristics of normal distribution, it is not sure that the generated normally distributed random numbers are all in the valid interval $([0,1]$ for porosity). Liu et al. [26] dealt with this problem by truncating some porosity values that are larger or smaller than the specified threshold values. Nevertheless, this truncation may destroy the property of the normal distribution. Additionally, the use of variance to quantitatively represent the heterogeneity of the porosity field is not commonly used in reservoir engineering. Here, we present a new initial porosity generation method to avoid the above problems. Noted that except the porosity obey the normal distribution, it has been shown that permeabilities in most reservoirs exhibit a log-normal distribution. Therefore, we first generate a log-normal distributed permeability field $K^{r}$, and then calculate the porosity field by solving the Carman-Kozeny equation Eq. (48).

The heterogeneity magnitude of the permeability field is quantitatively expressed using the Dykstra-Parsons coefficient $V_{D P}$, which is commonly used in the petroleum industry to measure the permeability variation[68], and is defined as

$$
V_{D P}=\frac{K_{0.5}-K_{0.16}}{K_{0.5}}
$$

where $K_{0.5}$ is the mean permeability, $K_{0.16}$ is the permeability that one standard deviation below the mean on a $\ln (K)$-probability plot. For the case that permeability is log-normal distributed, $V_{D P}$ can be related with the stand deviation of $\ln (K)$, i.e. $\sigma$ as

$$
V_{D P}=1-e^{-\sigma}
$$

The Dykstra-Parsons coefficient $V_{D P}$ ranges from 0 to 1 , and the larger the value of $V_{D P}$, the more heterogeneous of the reservoir. For most reservoirs, $V_{D P}$ falls within a range of $[0.5,0.9][69]$.

The moving average method proposed by Journel [70] is adopted here to generate a correlated permeability field. Firstly, a Gaussian distribution with zero mean and unit variance is generated. Then, the following steps are involved to achieve the correlation:

1. Select an appropriate type of covariance function to describe the correlation. Here we use the Gaussian covariance model as given by:

$$
C(r)=\sigma^{2} \exp \left(-\frac{r^{2}}{a^{2}}\right)
$$


where $\sigma^{2}$ is the variance, $a$ is the correlation length, $r$ is the space lag with $r=$ $\sqrt{x_{1}^{2}+x_{2}^{2}+\cdots+x_{n}^{2}}$ for $n$ dimension case.

2. Calculate the kernel matrix (weight matrix) $f(r)$ using the kernel of the square root of covariance formula, of which the analytical expression has been derived by Oliver [71]. The kernel of the square root of Gaussian covariance function used here is expressed as:

$$
f(r)=\sigma\left(\frac{4}{a^{2} \pi}\right)^{n / 4} \exp \left(-\frac{2 r^{2}}{a^{2}}\right)
$$

3. Convolve the previous generated Gaussian distribution $Z$ with the kernel matrix $f(r)$ to achieve the correlation as:

$$
Z^{\prime}=\int_{-\infty}^{\infty} f(r-s) Z(s) d s=f * Z
$$

Noted that the Fourier transform of the convolution of two functions is the product of their Fourier transforms, i.e.,

$$
\mathcal{F}(f * Z)=\mathcal{F}(f) \cdot \mathcal{F}(Z)
$$

Accordingly, a fast Fourier transform (FFT) algorithm and an inverse FFT algorithm are used to calculate the convolution in Eq. (53). Then, the correlated Gaussian distribution $Z^{\prime}$ is found to be

$$
Z^{\prime}=\mathcal{F}^{-1}(\mathcal{F}(f) \cdot \mathcal{F}(Z))
$$

4. Add the prior mean to the distribution $Z^{\prime}$ and then, calculate the exponential of the sum to get the log-normally distributed permeability field with specified mean and covariance. Mathematically this is written as

$$
K^{r}=\exp \left(\mu_{\text {prior }}+Z^{\prime}\right)
$$

The prior mean $\mu_{\text {prior }}$ and standard deviation $\sigma$ used in above procedures are given by

$$
\mu_{\text {prior }}=\ln K_{\text {avg }}-\frac{\sigma^{2}}{2}
$$

$$
\sigma=-\ln \left(1-V_{D P}\right)
$$

Eq. (57) is derived from the parametric relationship between the normal and lognormal distribution, and $K_{a v g}$ is the average permeability of the designed permeability field. Eq. (58) is the rearrangement of Eq. (50). 
The steps presented above are for generating an isotropic permeability field. Anisotropy can be achieved by stretching the axis of the coordinate system so that the spatial correlation in the new coordinate system is isotropic. The coordinate transformation is given by

$$
\left[\begin{array}{l}
x^{\prime} \\
y^{\prime} \\
z^{\prime}
\end{array}\right]=\left[\begin{array}{ccc}
1 & 0 & 0 \\
0 & \alpha_{x y} & 0 \\
0 & 0 & \alpha_{x z}
\end{array}\right]\left[\begin{array}{l}
x \\
y \\
z
\end{array}\right]
$$

with

$$
\alpha_{x y}=\frac{l_{x}}{l_{y}}, \alpha_{x z}=\frac{l_{x}}{l_{z}}
$$

where $l_{x}, l_{y}$, and $l_{z}$ are correlation length in $x, y$, and $z$ directions, respectively.

\section{Dimensionless model}

The non-dimensional forms of the equations presented in Section 2 are obtained by defining the following dimensionless parameters:

$$
\begin{gathered}
t_{D}=\frac{t}{L / u_{0}}, \mathbf{U}=\frac{\mathbf{u}}{u_{0}}, c_{D}=\frac{c_{f}}{c_{f 0}}, T_{D}=\frac{T}{T^{0}}, \\
\Lambda_{t}=\frac{K\left(K_{r w} / \mu_{w}+K_{r o} / \mu_{o}\right)}{K_{0}\left(K_{r w, 0 x}^{m a x} / \mu_{w}+K_{r, 0}^{m a x} / \mu_{o}\right)}, f_{w}=\frac{K_{r w} / \mu_{w}}{K_{r w} / \mu_{w}+K_{r o} / \mu_{o}}, \\
P_{D}=\frac{K_{0}\left(P-P_{e}\right)}{L u_{0}}\left(\frac{K_{r w, 0}^{m a x}}{\mu_{w}}+\frac{K_{r o, 0}^{m a x}}{\mu_{o}}\right), h_{T}^{2}=\frac{2 k_{s}\left(T^{0}\right) \overline{r_{0}}}{D_{m}^{0}}, D a=\frac{k_{s}\left(T^{0}\right) a_{v 0} L}{u_{0}}, \\
r_{p D}=\frac{r_{p}}{\overline{r_{0}}}, D_{m D}=\frac{D_{m}}{D_{m}^{0}}, a_{v D}=\frac{a_{v}}{a_{v 0}}, P e_{L}=\frac{u_{0} L}{D_{m}^{0}}, \eta=\frac{2 \overline{r_{0}}}{L}, H_{T}^{2}=\frac{k_{s}\left(T^{0}\right) a_{v 0} L^{2}}{D_{m}^{0}}, \\
\rho_{w D}=\frac{\rho_{w}}{\rho_{s}}, \rho_{o D}=\frac{\rho_{o}}{\rho_{s}}, \rho_{s D}=\frac{\rho_{s}}{\rho_{s}}=1, C_{w D}^{p}=\frac{C_{w}^{p}}{C_{s}^{p}}, C_{o D}^{p}=\frac{C_{o}^{p}}{C_{s}^{p}}, A_{E}=\frac{E_{a}}{R T^{0}}, \\
C_{s D}^{p}=\frac{C_{s}^{p}}{C_{s}^{p}}=1, P e_{H}=\frac{-\Delta H_{r} C_{s} C_{s}^{p}}{\phi S_{w} \Gamma_{e w}+\phi\left(1-S_{w}\right) \Gamma_{e o}+(1-\phi) \Gamma_{e s}}, H_{D}=\frac{\alpha c_{0}}{T_{0} \rho_{s} C_{s}^{p}}, N_{a c}=\frac{\alpha \rho_{s}}{\rho_{s}} .
\end{gathered}
$$

where the subscript $D$ indicates the corresponding variable is dimensionless; $L$ is the characteristic length, which takes the value of the core length for linear flow and the core radius for radial flow; $\mathbf{U}$ is the dimensionless velocity; $\Lambda_{t}$ is the dimensionless total mobility; $f_{w}$ is the fractional flow of water; $\eta$ is the pore-to-domain scale ratio; $A_{E}$ is the dimensionless activation energy; $H_{D}$ is the dimensionless exothermic heat of reaction; $h_{T}^{2}$ and $H_{T}^{2}$ are Thiele modulus defined on pore scale and core scale, respectively, and they are the ratio of reaction rate to diffusive mass transfer rate; $D a$ is the Damkohler number defined as the ratio of reaction rate to convective mass transport rate; $P e_{L}$ is the core scale Peclet number 
422

423

with

$$
\frac{\partial\left(\phi S_{w} c_{D}\right)}{\partial t_{D}}+\nabla \cdot\left(\mathbf{U}_{w} c_{D}\right)=\nabla \cdot\left(\mathbf{D}_{e D} \nabla c_{D}\right)-\frac{S h \cdot D_{m D} D a \cdot a_{v D} \cdot \exp \left(A_{E}\left(1-\frac{1}{T_{D}}\right)\right) c_{D}}{S h \cdot D_{m D}+h_{T}^{2} r_{p D} \cdot \exp \left(A_{E}\left(1-\frac{1}{T_{D}}\right)\right)}
$$

425

$$
\begin{aligned}
& \frac{\partial}{\partial t_{D}}\left[\left(\sum_{\alpha=w, o} \phi S_{\alpha} \rho_{\alpha D} C_{\alpha D}^{p}+(1-\phi)\right) \cdot T_{D}\right]+\sum_{\alpha=w, o} \rho_{\alpha} C_{\alpha D}^{p} \nabla \cdot\left(\mathbf{U}_{\alpha} T_{D}\right) \\
& =\frac{1}{P e_{H}} \nabla \cdot(\nabla T)+\frac{H_{D} S h \cdot D_{m D} D a \cdot a_{v D} \cdot \exp \left(A_{E}\left(1-\frac{1}{T_{D}}\right)\right) c_{D}}{S h \cdot D_{m D}+h_{T}^{2} r_{p D} \cdot \exp \left(A_{E}\left(1-\frac{1}{T_{D}}\right)\right)}
\end{aligned}
$$

$$
\frac{\partial \phi}{\partial t_{D}}=N_{a c} \cdot \frac{S h \cdot D_{m D} D a \cdot a_{v D} \cdot \exp \left(A_{E}\left(1-\frac{1}{T_{D}}\right)\right) c_{D}}{S h \cdot D_{m D}+h_{T}^{2} r_{p D} \cdot \exp \left(A_{E}\left(1-\frac{1}{T_{D}}\right)\right)}
$$

$$
\mathbf{D}_{e D}^{Y}=\frac{\phi S_{w} \alpha_{o s} D a D_{m D}}{H_{T}^{2}}+\lambda_{Y}\left|\mathbf{U}_{w}\right| r_{p D} \cdot \eta S_{w}
$$

The initial and boundary conditions can also be transformed into dimensionless form as

$$
-\Lambda_{t} \nabla P_{D} \cdot \mathbf{n}=1, \quad \text { on } \partial \Omega_{\text {in }}
$$




$$
S_{w}=1, \quad \text { on } \partial \Omega_{\text {in }}
$$

428

$$
c_{D}-\left(\frac{\phi S_{w} \alpha_{o s} D a D_{m D}}{H_{T}^{2}}+\lambda_{X}\left|\mathbf{U}_{w}\right| r_{p D} \cdot \eta S_{w}\right) \nabla c_{D} \cdot \mathbf{n}=1, \quad \text { on } \partial \Omega_{\text {in }}
$$

429

$$
\begin{gathered}
T_{D}=T_{f D}, \quad \text { on } \partial \Omega_{\text {in }} \\
P_{D}=0, \quad \text { on } \partial \Omega_{\text {out }}
\end{gathered}
$$

430

$$
\nabla c_{D} \cdot \mathbf{n}=0, \quad \text { on } \partial \Omega_{\text {out }} \text { on } \partial \Omega_{\text {tran }}
$$

431

$$
\nabla T_{D} \cdot \mathbf{n}=0 \quad \text { on } \partial \Omega_{\text {out }} \text { and } \partial \Omega_{\text {tran }}
$$

432

$$
\nabla P_{D} \cdot \mathbf{n}=0, \quad \text { on } \partial \Omega_{\operatorname{tran}}
$$

433

$$
c_{D}=0, T_{D}=T_{s D}, S_{w 0}=S_{w i, 0}, \quad \text { at } t=0
$$

\section{Numerical method}

The governing equations presented in Section 3 are a set of coupled, non-linear partial differential equations, which can be solved sequentially or using the global implicit method. The sequential method is adopted here to solve the coupled T-H-C problem because it possesses several attractive features, such as different numerical techniques can be used to solve the sub-problems, the size of the system of linear equations to be solved is not as larger as that involved in the global implicit method.

Using the sequential method, the flow equations are solved first to obtain the Darcy velocity of each phase. Then the calculated velocity field is substituted into the acid and energy transport equations to compute the concentration and temperature distribution. The mass balance equation of the rock, which describes the change of porosity with dissolution proceed, is coupled with the reactive-transport equation. Whereas the flow equations are solved using the fully implicit method, the equations governed the concentration and temperature transport are solved with the use of operator splitting method. 
The resulting nonlinear equations from the discretization of the flow equations are solved using the Newton-Raphson iteration method. The operator splitting is accomplished by splitting the reactive transport procedure into a physical transport process and a process incorporating reactions. In any single time step, the distribution of temperature and acid concentration governed by the physical transport are first calculated. Then their values are modified due to the reactions occur. Mathematically, this process starts by solving

$$
\left\{\begin{array}{l}
\frac{\partial\left(\phi S_{w} c_{D}\right)}{\partial t_{D}}+\nabla \cdot\left(\mathbf{U}_{w} c_{D}\right)=\nabla \cdot\left(\mathbf{D}_{e D} \nabla c_{D}\right) \\
\frac{\partial}{\partial t_{D}}\left[\left(\sum_{\alpha=w, o} \phi S_{\alpha} \rho_{\alpha D} C_{\alpha D}^{p}+(1-\phi)\right) \cdot T_{D}\right]+\sum_{\alpha=w, o} \rho_{\alpha} C_{\alpha D}^{p} \nabla \cdot\left(\mathbf{U}_{\alpha} T_{D}\right)=\frac{\nabla \cdot(\nabla T)}{P e_{H}} \\
\frac{\partial \phi}{\partial t_{D}}=0
\end{array}\right.
$$

to obtain the acid concentration and temperature at $t^{*}$, which is the time when the transport process has completed, but the reaction has not started.

Then the effect of reaction is included by solving

$$
\left\{\begin{array}{l}
\frac{\partial}{\partial t_{D}}\left(\phi S_{w} c_{D}+\frac{\phi}{N_{a c}}\right)=0 \\
\frac{\partial}{\partial t_{D}}\left[\left(\sum_{\alpha=w, o} \phi S_{\alpha} \rho_{\alpha D} C_{\alpha D}^{p}+(1-\phi)\right) \cdot T_{D}-\frac{H_{D} \phi}{N_{a c}}\right]=0 \\
\frac{\partial \phi}{\partial t_{D}}=N_{a c} \cdot \frac{S h \cdot D_{m D} D a \cdot a_{v D} \cdot \exp \left(A_{E}\left(1-\frac{1}{T_{D}}\right)\right) c_{D}}{S h \cdot D_{m D}+h_{T}^{2} r_{p D} \cdot \exp \left(A_{E}\left(1-\frac{1}{T_{D}}\right)\right)}
\end{array}\right.
$$

Discretizing Eq. (80) using the backward Euler approach, three coupled nonlinear equations are obtained for each node. Solving these equations by iterative method gives the acid concentration, temperature, and porosity values at $t+\Delta t$. Noted that the diffusion coefficient in Eq. (79) is also temperature dependent. Thus, iteration is needed between the transport and reaction process. After this iteration converges, the porosity value at time $t+\Delta t$ is used to update the parameters involved in the flow equations and then the algorithm marches forward.

The procedure described above continues until the acid breaks through the rock. For the single phase flow, the effective permeability of the porous media is related to the pressure drop through Darcy's law. Therefore, the pressure drop can be used to measure if the injected acid breaks out, which is widely used in previous works. However, for two-phase flow, the 
pressure drop will be affected by both the relative permeability and absolute permeability. In order to reduce the effect of relative permeability, at each time step, the effective permeability in the flow direction is calculated using the dynamic method. Specifically, a single flow problem is solved using the acidizing permeability field with the Dirichlet boundary condition imposed on the inlet and outlet boundaries and no-flow boundary condition imposed on other boundaries. After that, the pressure and velocity at each node can be obtained. Then, the effective permeability of the whole porous media can be calculated through

$$
K_{e f f}=\frac{\int_{\Omega} u(x, y, z) d V}{\int_{\Omega} \nabla P(x, y, z) d V}
$$

The acid breakthrough is defined as the increase in the effective permeability by a factor of 100 .

We use the finite volume method to discretize these governing equations. Specifically, the diffusion term is discretized via the central difference scheme. To avoid the instability issues and achieve the second-order accuracy in time, the transient term is discretized using the backward Euler scheme combined with an extrapolation technique as discussed in Maheshwari et al. [67]. For the convection term, in most of the previous relevant works, it is discretized using the upwind scheme. Even though the upwind scheme can guarantee unconditionally stable and leads to physically plausible predictions, it is not the ideal scheme because it is of low order and solutions obtained with this scheme are of low accuracy. Therefore, a high order high resolution scheme named MINMOD scheme is used in our work to discretize the convection terms $[72,73]$. The MINMOD scheme is constructed based on the Total Variation Diminishing (TVD) framework by express the value of the variable $\Psi$ at the cell face as

$$
\Psi_{f}=\Psi_{C}+\frac{1}{2} \psi\left(r_{f}\right)\left(\Psi_{D}-\Psi_{C}\right)
$$

with

$$
r_{f}=\frac{\Psi_{C}-\Psi_{U}}{\Psi_{D}-\Psi_{C}}
$$

and the limiter $\psi\left(r_{f}\right)$ subject to

$$
\psi\left(r_{f}\right)=\max \left(0, \min \left(1, r_{f}\right)\right)
$$

where the subscript $f$ represents the cell face; $D, C$, and $U$ denote the downstream, upstream, and far upstream nodes of the face $f$, respectively.

To show the necessity of using the high resolution scheme, the commonly used two-scale continuum model presented in [65] is resolved, with the discretization of the convection term 


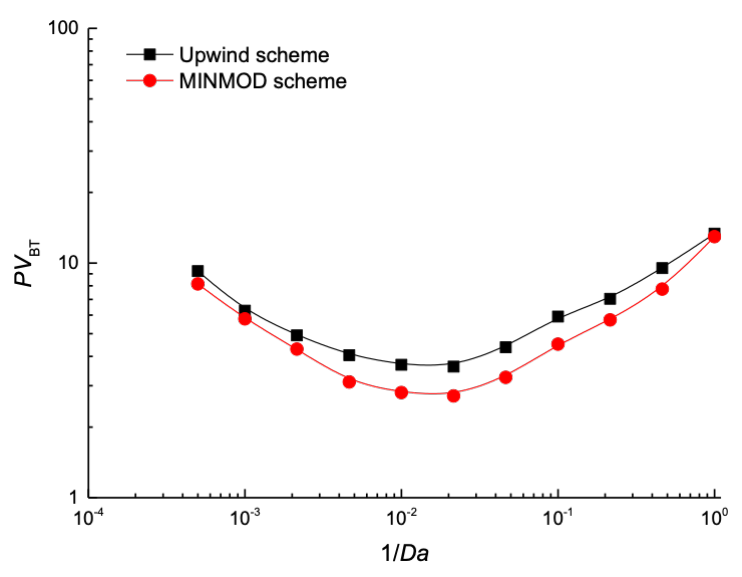

Figure 3: Comparison of the breakthrough volume calculated using the upwind scheme and the MINMOD scheme

using the upwind and MINMOD schemes, respectively. The $P V_{B T}$ computed by using these two schemes is compared in Fig. 3. It can be seen that the breakthrough volume calculated by using the upwind scheme is higher than that calculated using the MINMOD scheme. This is because that using upwind scheme to discretize the convection term will cause an additional component added to the diffusion coefficient of the equation being solved. This additional diffusion is come from the truncation error and is known as stream wise diffusion. As a result, the effect of diffusion on convection-diffusion transport is expanded. Specific to this example, the magnification of the diffusion coefficient leads to a result that more acid transports to the transverse direction resulting in more mineral to be dissolved, and consequently increase the amount of consumed acid. This is confirmed by comparing the dissolution patterns obtained through the use of these two discretization schemes. As shown in Fig. 4, the diameter of the dissolved channels calculated by using the upwind scheme is larger than that calculated by using the MINMOD scheme.

The grid size used in the simulation is determined based on the value of $P V_{B T}$ as described in [30]. Specifically, the grid is refined until the $P V_{B T}$ is insensitive to any grid size changes. During this process, very small time steps are used. To make the dissolution structures smoother, a finer grid is used in the current work. The calculations reported in Section 6 are performed with a mesh fixed at $80 \times 320$ ( 80 cells in $r$ direction and 320 cells in $\theta$ direction) for 2D radial cases, and $90 \times 240 \times 40$ (90 cells in $r$ direction, 240 cells in $\theta$ direction and 40 cells in $z$ direction) for 3D radial cases. It should be pointed out that the operator splitting method will result in mass-balance errors even though each sub-problem is solved exactly. To minimize this error, the CFL condition must always be satisfied when determining the time 
(a)
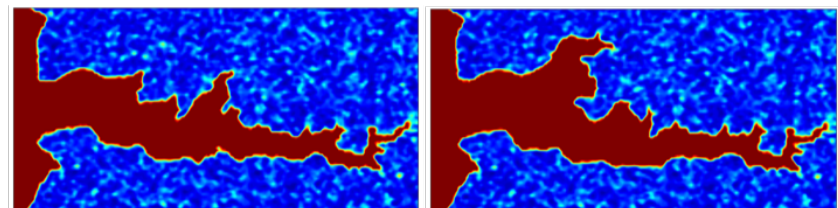

(b)
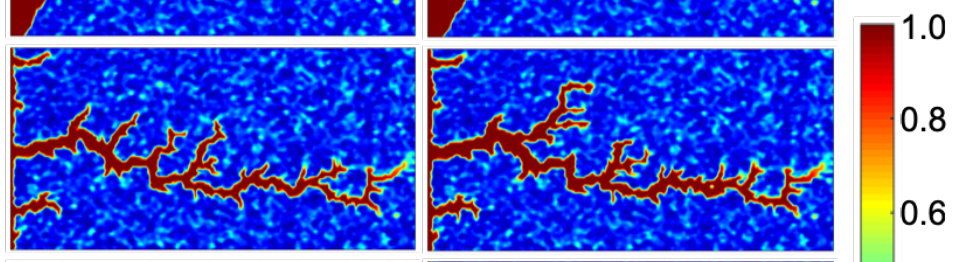

(c)

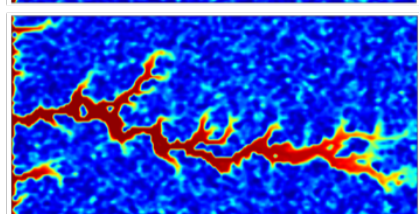

(d)

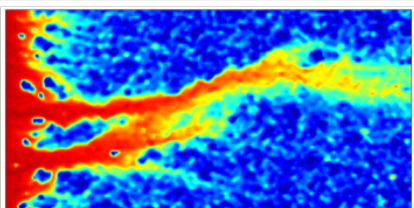

MINMOD

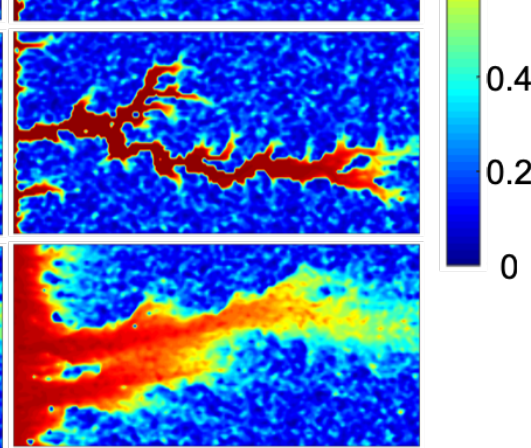

Upwind

Figure 4: Comparison of the dissolution patterns calculated using the upwind scheme and the MINMOD scheme

step. In our simulations, the dynamic time step scheme is used with the upper bound set by the CFL condition. In order to guarantee the stability and the accuracy of the simulation, the variation of the solution variables between any two consecutive time steps is set to be no more than a tolerance $\epsilon$. We use $S_{w}$ and $\phi$ to monitor the variation rate of the transport and reaction processes respectively. Specifically, we use the time step determined by the CFL condition as the initial time step, and if the difference in $S_{w}$ and $\phi$ calculated from two consecutive time steps is less than $\epsilon$, the current time step is used as the computational step, otherwise the time step is reduced until it satisfies the set criterion.

\section{Validation}

We have developed an in-house code based on the presented two-phase T-H-C coupled model with the use of the numerical method described above. The code is capable of simulating the acidizing process in carbonate rocks under 2-D/3-D linear/radial flow conditions. Because no numerical or experimental result that obtained considering all involved factors is available in the literature, we validate the code by reducing the model to a simple case. Considering that the T-H-C coupled problem is solved sequentially, the code will be validated if 
we can demonstrate that each sub-problem is solved correctly. On the other hand, it can be seen that the governing equation of the heat transport model has the same form as the acid transport equation and the same solution methodology is applied to them. Therefore, the code validation can be accomplished by verifying the correctness of (1) solving the two-phase flow problem, and (2) solving the reactive-transport problem. The former is performed by comparing the displacement front with the analytical solution while the latter is achieved by comparing the computed dissolution structures with the experimental observations.

To test the accuracy of our code in solving the two-phase flow problem, a 2-D Buckley-Leverett displacement problem is solved. The parameters used in this case are summarized in Table. 1. The relative permeability data is calculated through Eq. (10) and Eq. (11), and the resulting relative permeability curve is the same with that shown in Fig. 2(a). The capillary pressure and gravity terms are neglected, and the formation are assumed to be homogeneous. For the numerical case, we descretized the domain by a $100 \times 50$ orthogonal grid. Fig. 5 shows the saturation profiles of displacement after a 800 s and 1600s injection period. It can be seen that the numerical results is very close to the analytical solution.

Table 1: Values of parameters used in the Buckley-Leverett displacement problem

\begin{tabular}{ll}
\hline Parameters & Values \\
\hline Length of the domain, $L$ & $2 \mathrm{~m}$ \\
Width of the domain, $W$ & $1 \mathrm{~m}$ \\
Cross-section area, $A$ & $1 \mathrm{~m}^{2}$ \\
Permeability of the domain, $K_{B L}$ & $1 \times 10^{-12} \mathrm{~m}^{2}$ \\
Porosity of the domain, $\phi_{B L}$ & 0.2 \\
Water viscosity, $\mu_{w}^{B L}$ & $1 \times 10^{-3} \mathrm{~Pa} \cdot \mathrm{s}$ \\
Oil viscosity, $\mu_{o}^{B L}$ & $1 \times 10^{-3} \mathrm{~Pa} \cdot \mathrm{s}$ \\
Maximum relative permeability of water, $K_{r w, \text { max }}^{B L}$ & 0.26 \\
Maximum relative permeability of oil, $K_{r o, \text { max }}^{B L}$ & 0.98 \\
Irreducible water saturation, $S_{w i}^{B L}$ & 0.25 \\
Residual oil saturation, $S_{o r}^{B L}$ & 0.22 \\
Corey oil exponent, $n_{o}^{B L}$ & 0.24 \\
Corey water exponent, $n_{w}^{B L}$ & 0.24 \\
Injection rate, $q_{t}$ & $1 \times 10^{-4} \mathrm{~m}^{3} / \mathrm{s}$ \\
\hline
\end{tabular}

To test the effectiveness of the code in solving the reactive-transport problem, the pre- 


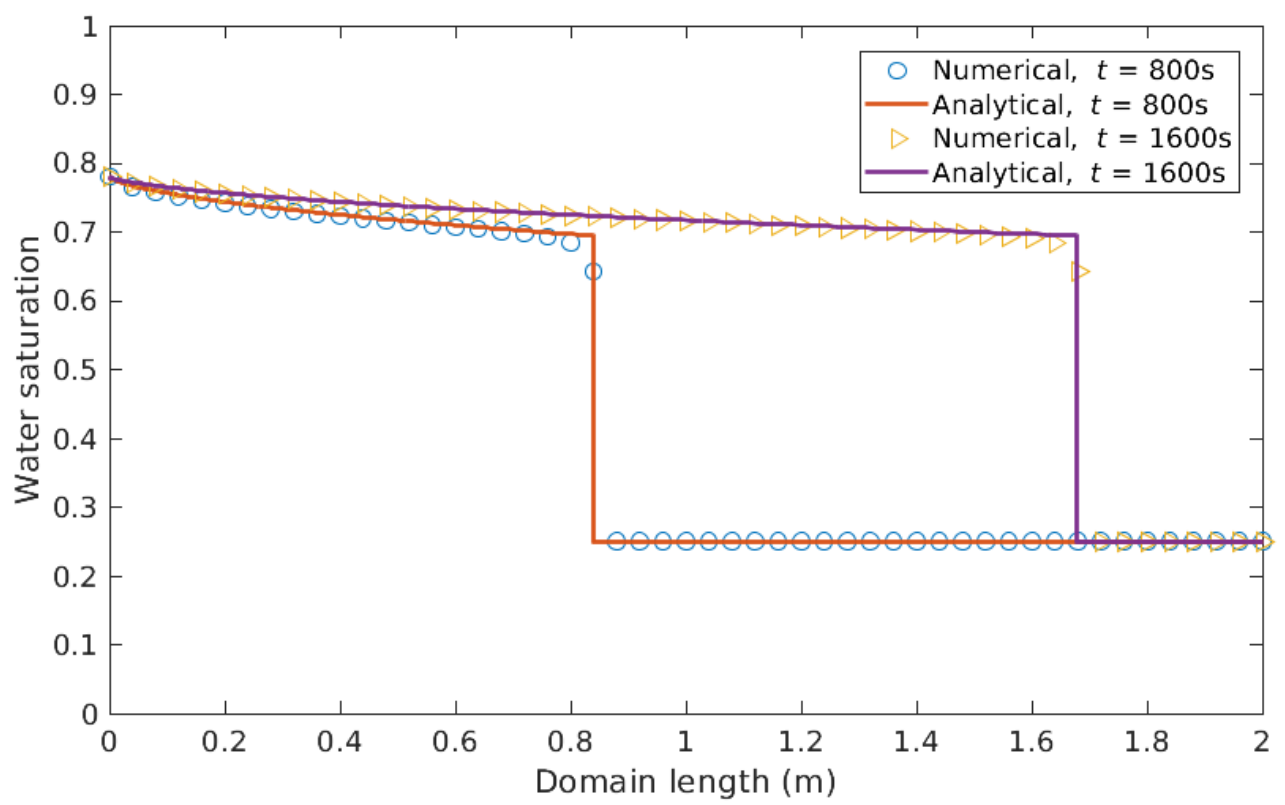

Figure 5: Comparison of the water saturation profiles from numerical solution and analytic solution

sented two-phase T-H-C coupled model can be reduced into the TSC model by neglecting the effect of heat transfer and using the single-phase flow field. The accurateness of the code in solving the reactive-transport problem under radial conditions has been validated in our previous works [25]. Here we test the code by comparing the simulation results with the experimental observations reported by McDuff et al. [74] to show its capacity for solving the reactive-transport problem under the 3-D linear flow condition. In McDuff's acidizing experiment, the $\mathrm{HCl}$ is injected into a series of carbonate cores with a length of $15.2 \mathrm{~cm}$ and a diameter of $3.8 \mathrm{~cm}$. Through high resolution CT scanning of acidified cores, the corresponding 3-D visualization images of dissolution patterns at different injection rates were obtained, as shown in Fig. 6(B). We use our numerical model to replicate the experimental operating conditions. The value of parameters used in the simulation is same with the data used in the experiment. Because some variables involved in the numerical model, such as the average pore radius, the interfacial surface area, the diffusion coefficient, etc., are not specified in the experiment report, we evaluate their values via previous similar acidizing numerical studies $[67,41]$. The dissolution patterns calculated using our numerical simulator for different injection rates are depicted in Fig. 6(A). It can be seen that these dissolution patterns generating at low, medium, and high injection rates are all in good agreement with that observed in the acid flooding experiment. This indicates that the code we developed is effective in solving the reactive-transport problem. 
(a)
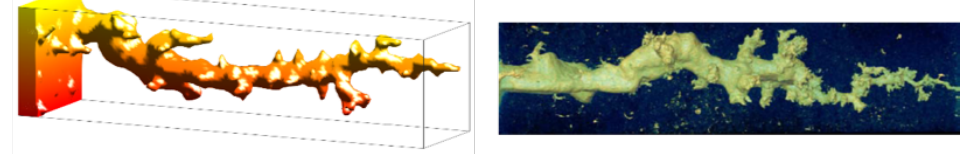

(b)
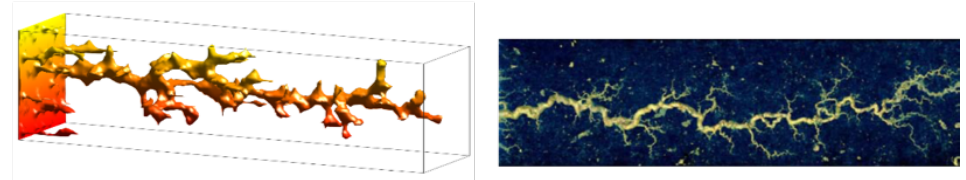

(c)

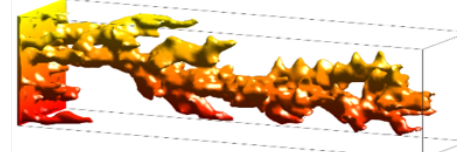

(A)

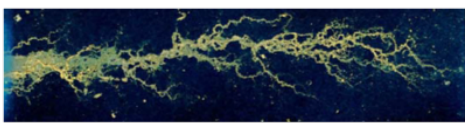

(B)

Figure 6: Comparison of (A) the dissolution patterns calculated using our code with (B) the experimental observation [74] for (a) low injection rate, (b) medium injection rate, and (c) high injection rate.

\section{Results and analysis}

This section presents simulation results to analyze the sensitivity of the dissolution dynamic with respect to the rock properties, temperature, and two-phase flow. The values of variables used in the simulations are shown in Table 2. All the values remained the same throughout the study unless otherwise stated.

Table 2: List of values of parameters used in simulation

\begin{tabular}{ll}
\hline Parameters & Values \\
\hline Initial irreducible water saturation, $S_{w i, 0}$ & 0.25 \\
Initial residual oil saturation, $S_{o r, 0}, 0.22$ \\
Initial maximum relative permeability of aqueous phase, $K_{r w, 0}^{\max 0}$ & 0.26 \\
Initial maximum relative permeability of oil-phase, $K_{r o, 0}^{\max }$ & 0.98 \\
Water density, $\rho_{w}$ & $1100 \mathrm{~kg} / \mathrm{m}^{3}$ \\
Water viscosity, $\mu_{w}$ & $1 \mathrm{mPa} \cdot \mathrm{s}$ \\
Specific heat capacity of water, $C_{w}^{p}$ & $4130 \mathrm{~J} /(\mathrm{kg} \cdot \mathrm{K})$ \\
Thermal conductivity of water, $\Gamma_{w}$ & $0.6 \mathrm{~J} /(\mathrm{s} \cdot \mathrm{cm} \cdot \mathrm{K})$ \\
Oil density, $\rho_{o}$ & $1000 \mathrm{~kg} / \mathrm{m}^{3}$ \\
Oil viscosity, $\mu_{o}$ & $1 \mathrm{mPa} \cdot \mathrm{s}$ \\
Specific heat capacity of oil, $C_{o}^{p}$ & $1200 \mathrm{~J} /(\mathrm{kg} \cdot \mathrm{K})$
\end{tabular}


Thermal conductivity of oil, $\Gamma_{o}$

3-D radial core dimensions, $r_{e}, r_{w}, h$

2 -D radial core dimensions, $r_{e}, r_{w}$

Initial average porosity, $\phi_{0}$

Initial interfacial area per unit volume of rock, $a_{v 0}$

Initial average permeability, $K_{0}$

Initial average pore radius, $\overline{r_{0}}$

Initial water saturation, $S_{w 0}$

Rock density, $\rho_{s}$

Specific heat capacity of rock, $C_{s}^{p}$

Thermal conductivity of rock, $\Gamma_{s}$

Reaction rate constant, $k_{s}^{0}$

Diffusion coefficient at $T^{0}, D_{m}^{0}$

Activation energy, $E_{a}$

Activation energy of diffusion, $E_{D}$

Universal gas constant, $R$

Acid capacity number $N_{a c}$

Temperature of the rock, $T_{s}$

Temperature of the acid at the inlet, $T_{f}$

Pressure at the exit, $P_{e}$

Inlet acid concentration, $c_{f 0}$

Constants in Eqs. (12) (15): $a_{w}, a_{o}, a_{k w}, a_{k o}$

Asymptotic Sherwood number, $S h_{\infty}$
$0.8 \mathrm{~J} /(\mathrm{s} \cdot \mathrm{cm} \cdot \mathrm{K})$

$10 \mathrm{~cm}, 1 \mathrm{~cm}, 4 \mathrm{~cm}$

$5 \mathrm{~cm}, 1 \mathrm{~cm}$

0.3

$50 \mathrm{~cm}^{-1}$

$2 \mathrm{md}$

$1 \times 10^{-4} \mathrm{~cm}$

0.251

$2600 \mathrm{~kg} / \mathrm{m}^{3}$

$879 \mathrm{~J} /(\mathrm{kg} \cdot \mathrm{K})$

$1.57 \mathrm{~J} /(\mathrm{s} \cdot \mathrm{cm} \cdot \mathrm{K})$

$0.2 \mathrm{~cm} / \mathrm{s}$

$3.6 \times 10^{-5} \mathrm{~cm}^{2} / \mathrm{s}$

$50241 \mathrm{~J} / \mathrm{mol}$

$16700 \mathrm{~J} / \mathrm{mol}$

$8.314 \mathrm{~J} /(\mathrm{mol} \cdot \mathrm{K})$

0.11

$343 \mathrm{~K}$

$298 \mathrm{~K}$

$20 \mathrm{MPa}$

2 wt $\% \mathrm{HCl}$

$10,10,10,10$

3

\subsection{Effect of rock properties}

\subsubsection{Effect of heterogeneity magnitude}

Carbonate rocks are inherently heterogeneous in permeability, porosity, and mineralogical distribution. This heterogeneity comes from the evolution of the rock over a long period. It is difficult to experimentally study the effect of rock heterogeneity on the dissolution dynamics of acidization, because it is not possible to get natural rock samples with different heterogeneity magnitude but identical other properties. On the contrary, this effect can be easily investigated numerically by varying the magnitude of the heterogeneity while keeping other variables fixed. Some efforts have been made to do this. De Oliveira et al. [75] studied 
the effect of mineralogical heterogeneity and found that it influences both the wormhole structure and $P V_{B T}$. Kalia and Balakotaiah [65], Maheshwari, et al. [67, 76], and Liu, et al [25] discussed the effect of porosity heterogeneity on dissolution process by performing simulations of carbonate acidizing under 2-D linear, 3-D linear, and 3-D radial flow conditions. However, the effect of permeability heterogeneity on dissolution patterns and breakthrough volume is still remained to be investigated.

Here, with the use of the Dykstra-Parsons coefficient $V_{D P}$, we analyze the effect of permeability heterogeneity magnitude on the dissolution process under 3-D radial condition. In order to avoid complications from heat transfer and multi-phase flow, the following parameter values are set as:

$$
T_{f}=T_{s}=343 \mathrm{~K} ; S_{w 0}=1 ; K_{r w}=1 ; K_{r o}=0 .
$$

The influence of varying $V_{D P}$ on $P V_{B T}$ for different acid injection rate (presented in terms of $D a$ ) is plotted in Fig. 7. It can be seen that when the injection rate is very low $\left(1 / D a=5 \times 10^{-4}\right)$ or very high $(D a=1.3), P V_{B T}$ keeps nearly as a constant with the increase of $V_{D P}$. The cause for this behavior can be understood by insightfully examining the mechanism that controls the dissolution dynamic. When the acid is injected into the rock at a very low rate, the transport velocity of the solute is very slow compared to the reaction rate, which results in that most of the acid is consumed instantaneously at the inlet before it penetrates deep into the rock. In this case, as discussed by Kalia [23, 65], the breakthrough volume is determined by the acid dissolution capacity and the initial average porosity, so it is independent with the heterogeneity magnitude. On the other hand, when the injection rate is very high, the injected acid transports so fast that it does not have sufficient time to react with the rock. As a result, all the connected pores are filled with the acid leading to a uniform increase in porosity. In this case, the breakthrough volume depends only on the injection rate, acid dissolution capacity, and the initial concentration of the injected acid, as analyzed by Kalia [23]. Therefore, the increase in the heterogeneity magnitude does not influence on $P V_{B T}$.

The conclusion obtained from the simulation with high injection rate is slightly different from that presented by Kilia [65], in which a rapid decline in $P V_{B T}$ is observed when the heterogeneity magnitude of the porosity field is extremely high. It should be pointed out that the initial porosity field used in [65] for performing the numerical experiments is uniformly distributed in a given interval $\left[\phi_{0}-\Delta \phi_{0}, \phi_{0}+\Delta \phi_{0}\right]$, and the heterogeneity magnitude of this porosity field is defined by the value of $\Delta \phi_{0}$. However, it is not a sensible parameter 


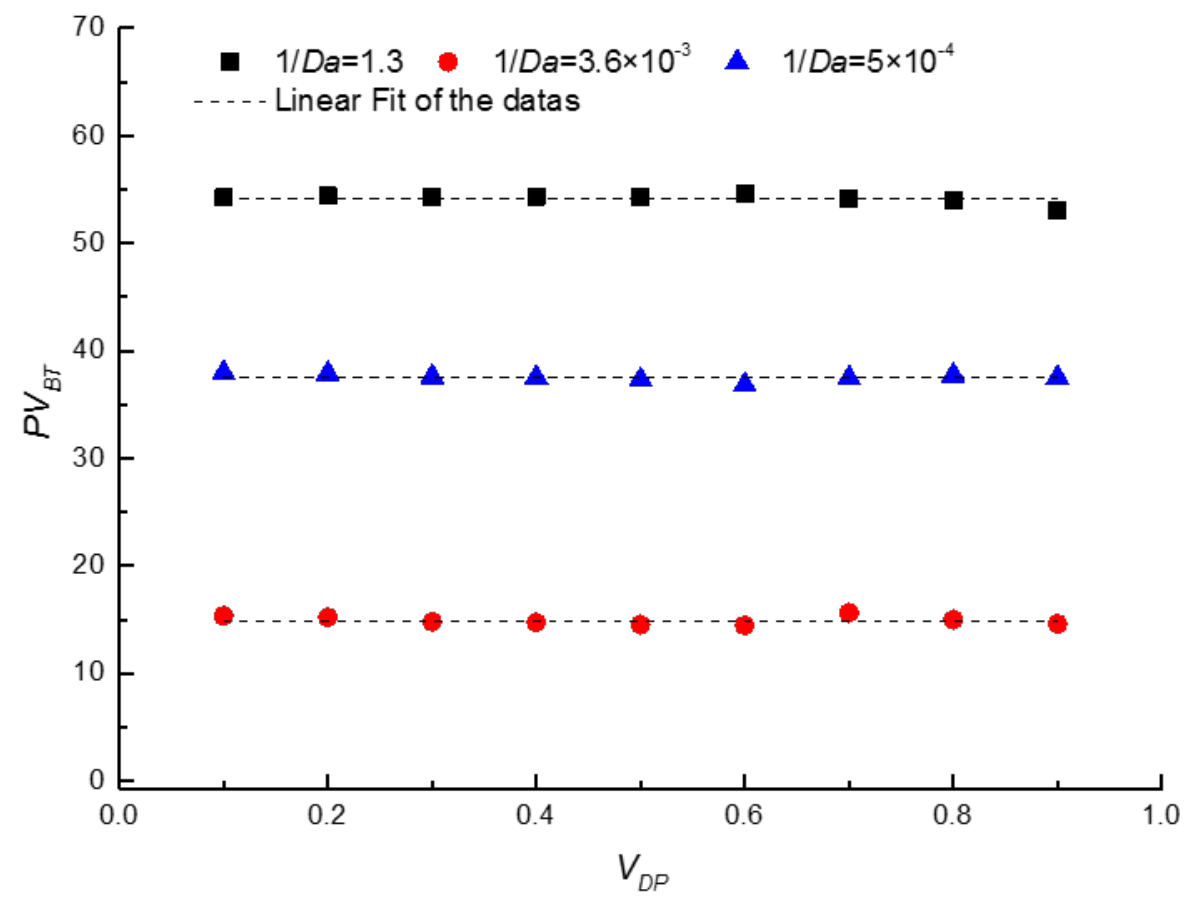

Figure 7: $P V_{B T}$ as a function of $V_{D P}$ for very high and very low $D a$ values

to quantitatively analyze the change of $P V_{B T}$ with heterogeneity magnitude because the value of $\Delta \phi_{0}$ is not independent. For example, based on Kilia's definition, the heterogeneity magnitude value of porosity fields distributed in both $[0.2-0.19,0.2+0.19]$ and $[0.5-$ $0.19,0.5+0.19]$ is 0.19 , but the actual heterogeneity magnitude is different. For the former, $\Delta \phi_{0}=0.19$ stands for a very high heterogeneity magnitude, while for the later, $\Delta \phi_{0}=0.19$ means the porosity field has an intermediate heterogeneity magnitude. The decline in $P V_{B T}$ observed by Kilia[65] may because the porous medium generated when $\Delta \phi_{0}$ approaches $\phi_{0}$ will contain more and more regions that are almost impermeable.

When acid is injected at the optimum rate, the effect of $V_{D P}$ on $P V_{B T}$ is quite different from that observed at very low and very high injection rates, as shown in Fig. 8. A minimum is observed in $P V_{B T}$ indicating a critical heterogeneity magnitude ( $V_{D P}=0.8$ in this case), which confirms the studies by $[65,67,76,25]$. This phenomenon can be seen more clearly in Fig. 9, where the wormhole structures at the breakthrough time are displayed for various heterogeneity magnitudes. When the heterogeneity magnitude is very low, the permeability over all spatial directions is almost a constant, so it is difficult for the injected acid to find a preferential way to flow out. As a result, a considerable number of straight wormholes with low branching are formed when acid breaks through the rock, as shown in Fig. 9(a). 
Therefore, a high volume of acid is needed to dissolve the rock to form these wormholes. As the heterogeneity magnitude increasing, the number of dominate wormholes decreases and the formed wormholes become highly branched, as shown in Fig. 9 (b) and (c). This is because that the presence of some high permeability regions makes acid easy to flow in. When the heterogeneity magnitude reached at the critical value $\left(V_{D P}=0.8\right.$ in our simulations, as shown in Fig. 9(d)), only one dominate wormhole is formed and the growth of others is stopped because the one providing the least resistance to flow captures most of the acid. As the heterogeneity magnitude continues to increase, more high permeability regions exist in the rock leading to some highly branched wormholes as can be seen in Fig. 9(f). Besides, the acid must dissolve some rocks with lower porosity to connect these high permeability regions. Thus, acid dissolves overall more rock in this case than the rock with critical heterogeneity, which is responsible for the resulting higher $P V_{B T}$ value.

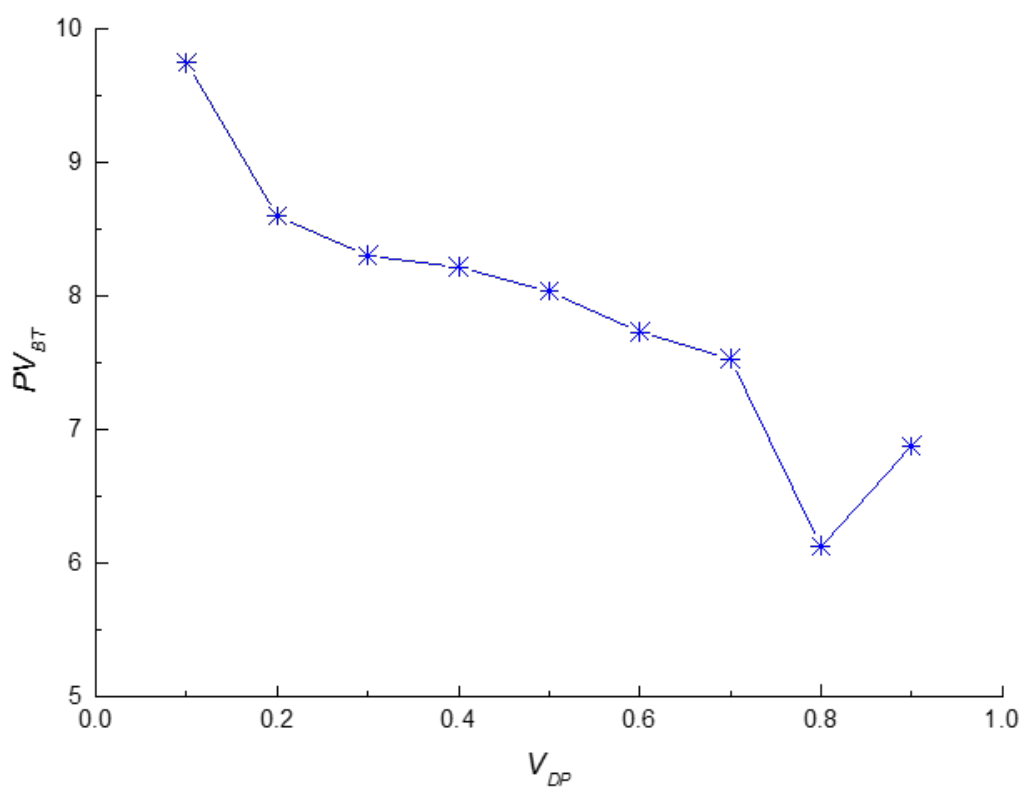

Figure 8: Effect of heterogeneity magnitude $V_{D P}$ on the breakthrough volume $P V_{B T}$ at the wormhole regime $(1 / D a=0.006)$

The simulations for varying $V_{D P}$ is also performed under 2-D radial condition, and the wormhole structures obtained at optimum injection rate are displayed in Fig. 10. Comparing Fig. 10 with Fig. 9, it can be clearly seen that the main difference between the 2-D and 3-D analysis is the number of wormholes and side branches. In the following analysis, because we are only interested in qualitative understanding of the effects of specifying parameters on acidizing process, the 2-D simulations are sufficient to provide this insight. Moreover, 

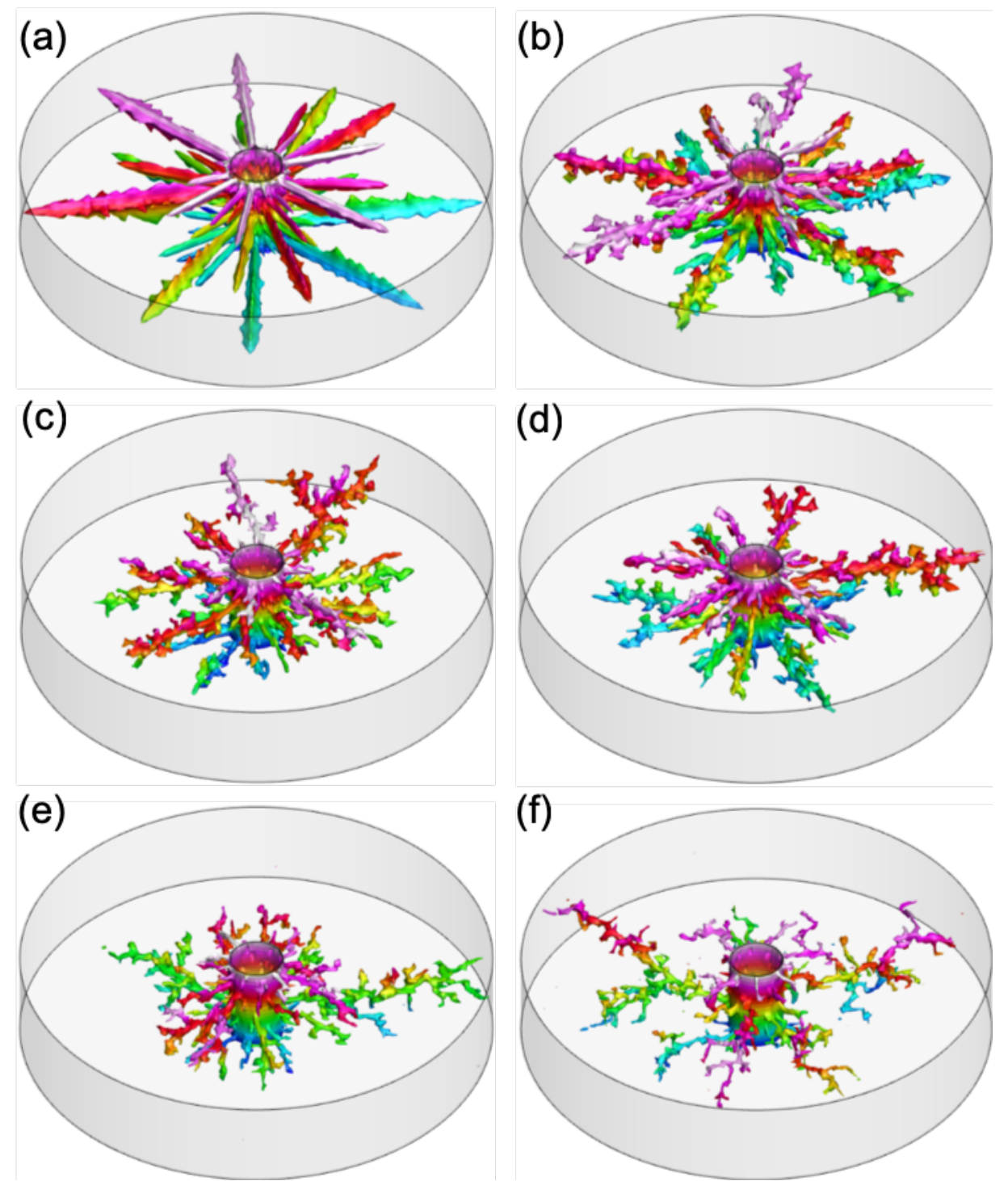

Figure 9: The effect of heterogeneity magnitude on wormhole structures under 3-D condition: (a) $V_{D P}=0.1$, (b) $V_{D P}=0.5$, (c) $V_{D P}=0.6$, (d) $V_{D P}=0.7$, (e) $V_{D P}=0.8$, (f) $V_{D P}=0.9$. (The color is to show the elevation.) 
the simulation under 2-D conditions is more time-saving than that under 3-D conditions, and the visualization of the temperature and saturation distribution in 2-D is easier than in 3 -D. Therefore, in the following sections, we use a 2-D radial geometry to perform the simulation and analyze the effect of various parameters.
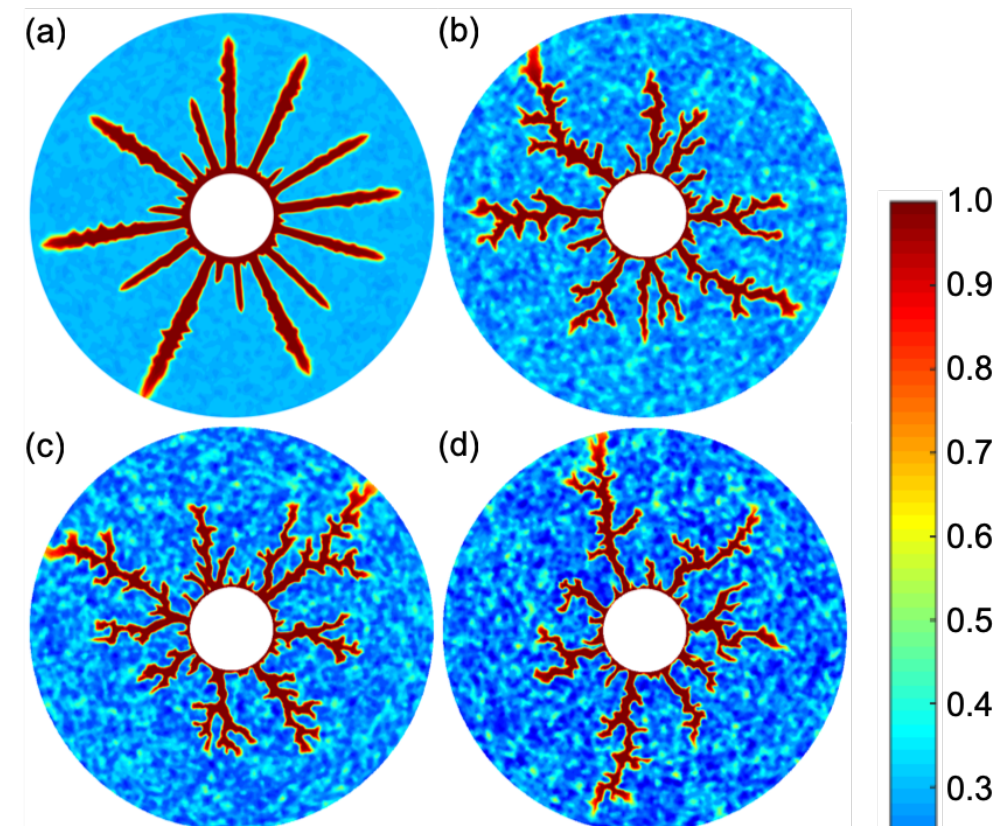

(d)
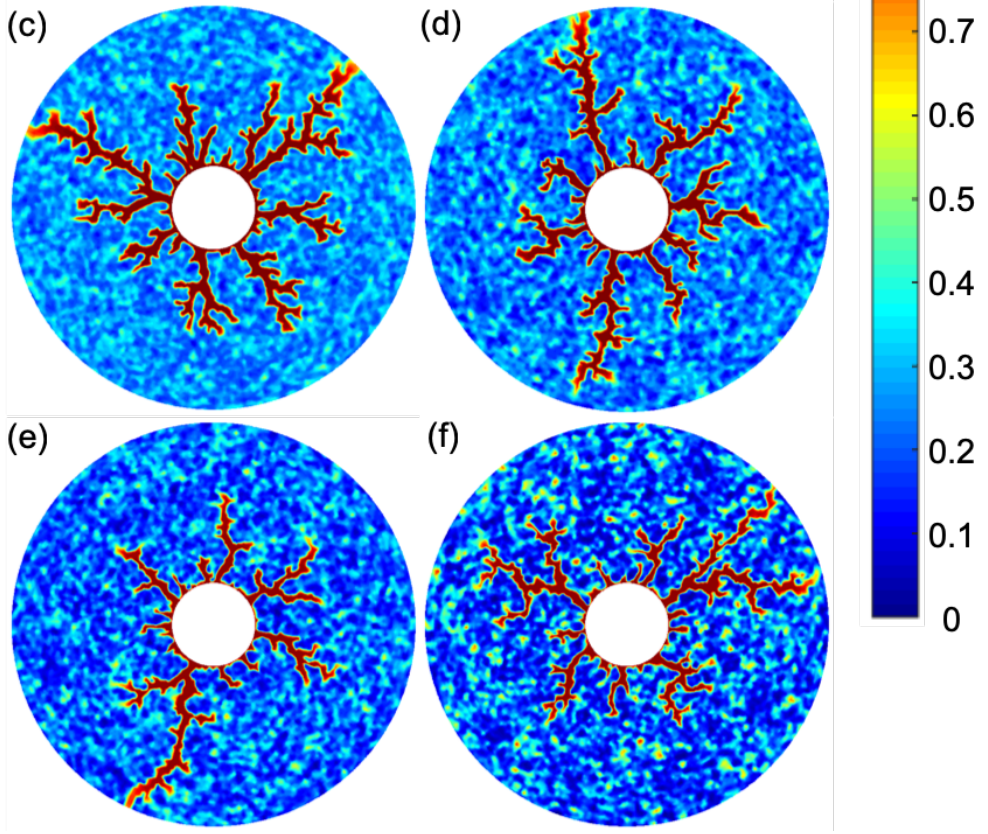

Figure 10: The effect of heterogeneity magnitude on wormhole structures under 2-D condition: (a) $V_{D P}=$ 0.1, (b) $V_{D P}=0.5$, (c) $V_{D P}=0.6$, (d) $V_{D P}=0.7$, (e) $V_{D P}=0.8$, (f) $V_{D P}=0.9$.

\subsubsection{Effect of anisotropy}

The effect of anisotropy is studied by varying the ratio of correlation length in $x$ and $y$ directions. Four porosity fields with different correlation length ratio of value $l_{x} / l_{y}=1$, $l_{x} / l_{y}=2, l_{x} / l_{y}=5$, and $l_{x} / l_{y}=10$, are generated. The heterogeneity magnitude for these cases is fixed at $V_{D P}=0.75$. The acid is injected at the optimum rate. Fig. 11 depicts the wormhole structures when acid breaks through the core. As intuitively expected, with 
the increase of anisotropic magnitude, wormhole propagation becomes more and more directional. For the isotropic porous media (Fig.11(a)), the generated wormholes are distributed uniformly and symmetrically in terms of angular direction. As the ratio of correlation length in horizontal and vertical directions increasing, the propagation of wormholes in the vertical direction is suppressed. This is because the injected acid mainly flows along the path with the least resistance. The pores have better connectivity in the horizontal direction than in the vertical direction leading to lower resistance for fluid flowing. When the anisotropic magnitude is very high, as shown in Fig. 11 (d), the growth of wormhole in the vertical direction will be stopped and wormholes are only formed in the direction that has a larger correlation length. It should be noted that the main purpose of injecting acid into a carbonate reservoir is to ensure that all reservoir zones are sufficiently treated, not limited to wormhole formation. Accordingly, for the anisotropic formation, self-diverting acids are recommended to make the stimulation more uniform. The simulation study of injecting self-diverting acid into carbonate rocks using the two-phase coupled T-H-C model will be conducted in our future work.

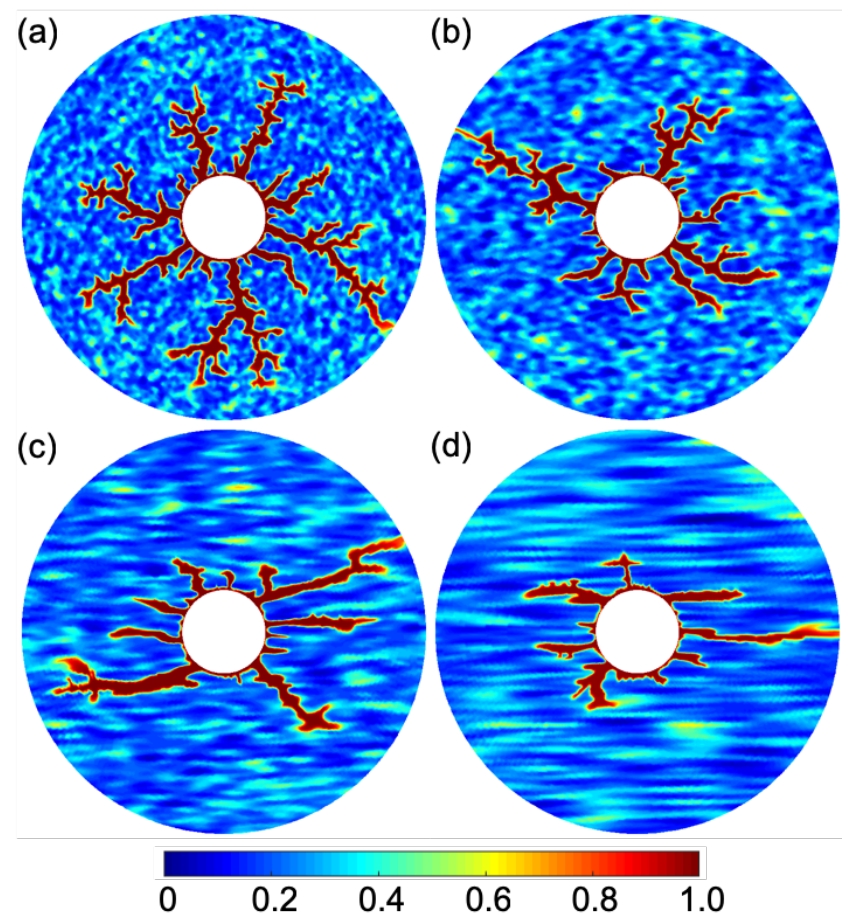

Figure 11: The effect of anisotropy magnitude on wormhole structures 


\subsection{Effect of reaction exotherm}

Temperature plays a significant role in carbonate acidizing treatments, in which the acid is typically injected at a temperature below the reservoir temperature. During the process, temperature changes occur inside both the wellbore and formation. In the wellbore, the temperature of injected acid increase with the depth increasing because of the existence of the geothermal gradient. In the formation, temperature changes with space and time are attributed to (1) the heat transfer between injected acid and formation, and (2) the exothermic heat of reaction. The effect of heat transfer on the optimum injection rate, $P V_{B T}$, and dissolution patterns has been investigated experimentally and numerically in previous works[3, 18, 32, 33, 34], and some remarkable observations have been reported. For example, Fredd [3] injected different types of acid into limestone rocks at varying temperatures and found that the optimum injection rate increases with the increase of temperature, but the $P V_{B T}$ may decrease or increase with the increase of temperature depending on the acid types. A similar trend is observed by Kalia [33] through numerical simulation. Thus, we are not going to repeat this kind of studies here but focus on the effect of reaction exotherm.

By measuring the temperature along the wellbore during a real acidizing treatment [33], it has been found that the temperature at some points is higher than the baseline temperature. This increase in temperature is apparently the result of the exothermic heat of reaction. Therefore, understanding the effect of reaction exotherm on dissolution dynamic is critical for understanding the stimulation effect for real acidizing treatment. However, only limited efforts are made in this regard[33, 34]. Kalia [33] investigated the effect of exothermic heat by including and not including the energy transport equation in the model when simulating the wormhole formation, and concluded that the temperature increase resulting from the exothermic heat of reaction does not affect the $P V_{B T}$ value under radial flow condition. Although this conclusion obtained by their work is valid for the wormhole regime, they did not study the effect of a wider range of injection rates. Here, the influence of reaction exotherm is investigated in more detail by simulating the acidizing process at a wider range of injection rates.

In our numerical experiment, the acid is injected into the rock radially at the same temperature $(343 \mathrm{~K})$ with the formation. The energy balance equation is included in the model to simulate the generation and conduction of the exothermic heat of reaction. So, the reactive-transport phenomena occur under non-isothermal conditions. Again, in order to avoid complications from the multi-phase flow, the initial water saturation is set to 1 . The calculated $P V_{B T}$ is plotted against $1 / D a$ in Fig. 12, and is compared with the isother- 


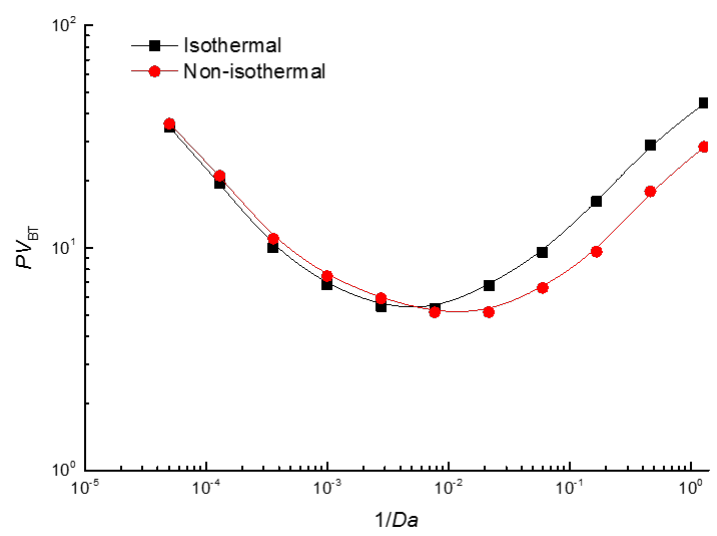

Figure 12: Comparison of the $P V_{B T}$ calculated under the isothermal and non-isothermal conditions

mal case, in which the energy balances are not included. It can be seen from Fig. 12, at the intermediate injection rate $(1 / D a=0.008)$ where the wormhole is formed, $P V_{B T}$ obtained under the non-isothermal condition is almost the same with that obtained under the isothermal condition, which confirms Kalia's observation [33]. However, at low injection rates where conical wormhole is formed, $P V_{B T}$ for the non-isothermal case is higher than the isothermal case. The governing mechanism of dissolution patterns is responsible for this difference in $P V_{B T}$. As acid is injected into the rock, the reaction occurs and leads to an increase in temperature due to the exothermic heat. This increase in temperature results in an increase in diffusivity and reactivity. When the injection rate is low, the reaction process is controlled by the solute transport rate because it is lower than the reaction rate. Whereas the increase in reaction rate will not affect the relative magnitude of them, the increase in diffusivity causes more acid to transport to the surrounding pores before being consumed. As a result, the diameter of the dissolved channels obtained under non-isothermal conditions is larger than that obtained under isothermal conditions, which leads to a high $P V_{B T}$ value for the non-isothermal case. This is evident in Fig. 13 where the dissolution structures for both non-isothermal and isothermal cases at different injection rates are displayed. On the other side, when the injection rate is high, $P V_{B T}$ for the non-isothermal case is lower than the isothermal case as shown in Fig. 12. This is because at high injection rate the velocity of solute transport is higher than the reaction rate. With the increase in reaction rate as a result of the reaction exotherms, more acid is consumed before penetrating further, which retards the formation of the ramified wormholes as can be seen in Fig. 13. For the case that the injection rate has the value of $1 / D a=0.06$, the wormhole simulated under the isothermal condition is ramified while the branchiness of wormhole calculated under the 
non-isothermal condition is low (Fig. 13 row (d)). The temperature distributions for the non-isothermal case at the early time and the breakthrough time are shown in Fig. 14. It can be observed that the exothermic heat is released at the dissolution front and is flushed into the formation. When acid breaks through the rock, although the dissolved channels are cooled down by the subsequent acid, the temperature in undissolved regions is higher than its initial temperature.

\subsection{Effect of two-phase flow}

\subsubsection{Effect of relative permeability curve}

As presented in the previous section, we have developed a model to describe the evolution of relative permeability curve with porosity. In this model, a constant $a$ was used to describe the change in $S_{w i}, S_{o r}, K_{r o}^{\max }$, and $K_{r w}^{\max }$ with porosity. Because the value of $a$ is difficult to determine, here we analyze the effect of the choice of $a$ value on the dissolution dynamic. Four simulation cases with the value of $a=10^{-4}, a=1, a=5$, and $a=100$ are performed and the computational results are compared with that calculated using a fixed relative permeability curve.

The acid is injected at the optimum rate so that wormhole dissolution is formed. The breakthrough volumes for these cases are compared in Fig. 15 (f). It can be seen that the $P V_{B T}$ calculated using a fixed relative permeability cure is larger than that using our developed model, but $P V_{B T}$ is almost insensitive to the value of $a$. As dissolution proceeding, if the rock is completely dissolved, no fluid will be trapped in pores. Accordingly, the relative permeability of both acid and oil phase inside wormholes approaches 1 , which results in that almost all the oil in the wormholes was displaced by acid. So, the acid should be transported more effectively in wormholes than in other areas, but using a fixed relative permeability curve ignored this effect. The transport efficiency of acid in the wormhole is underestimated with the use of a fixed relative permeability curve, which leads to the high breakthrough volume calculated. This can be seen clearly in Fig. 15 (a) (e), where the saturation distributions at the breakthrough time are displayed for various $a$ values and compared with that obtained using a fixed relative permeability curve.

\subsubsection{Effect of oil-water viscosity ratio}

The influence of varying the oil viscosity under isotherm condition has been studied by Wei [36] and Mahmoodi [37]. A common conclusion that $P V_{B T}$ decreases with the increase of oil viscosity was obtained by them. However, the reason why this decrease occurs was explained differently. Whereas Wei [36] claim that larger oil viscosity leads to a decrease in 
(a)

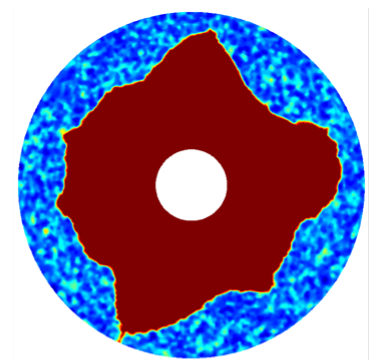

(b)

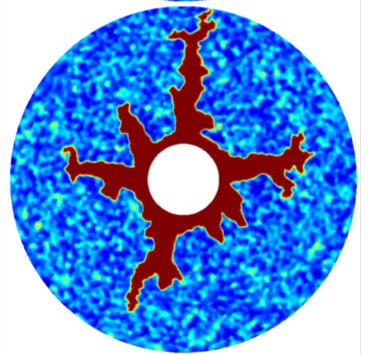

(c)

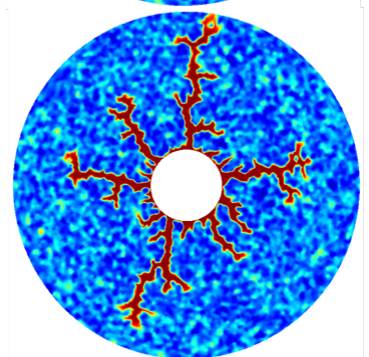

(d)

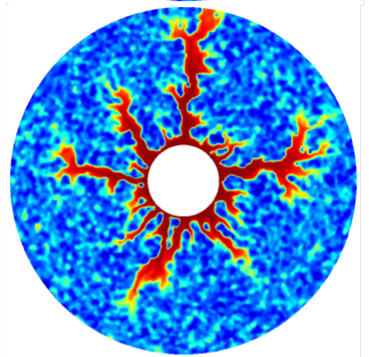

(e)

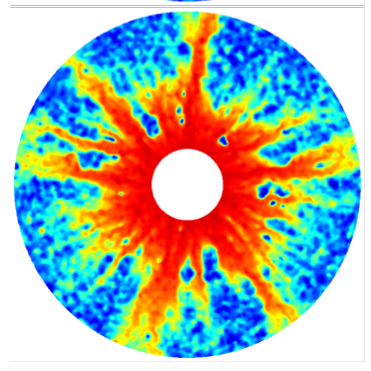

Isothermal
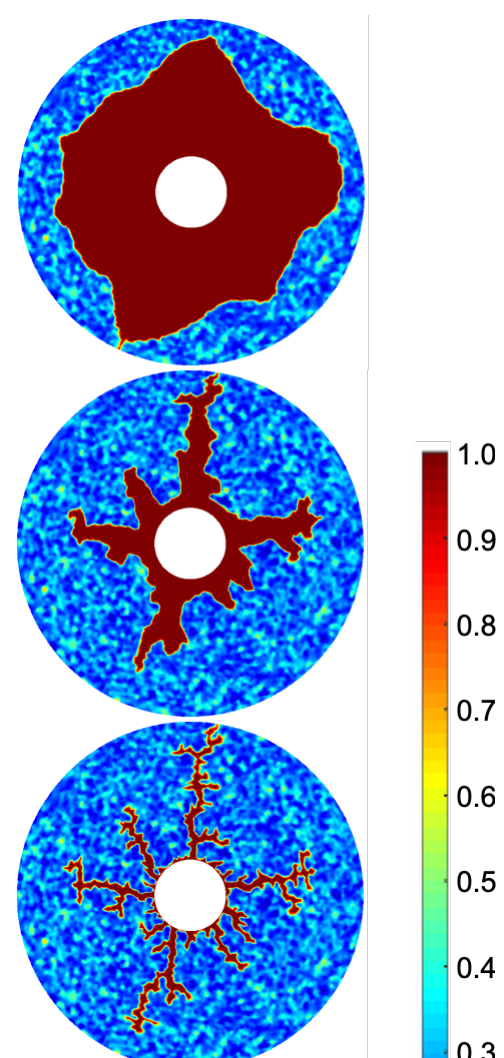

0.3

0.2

0.1
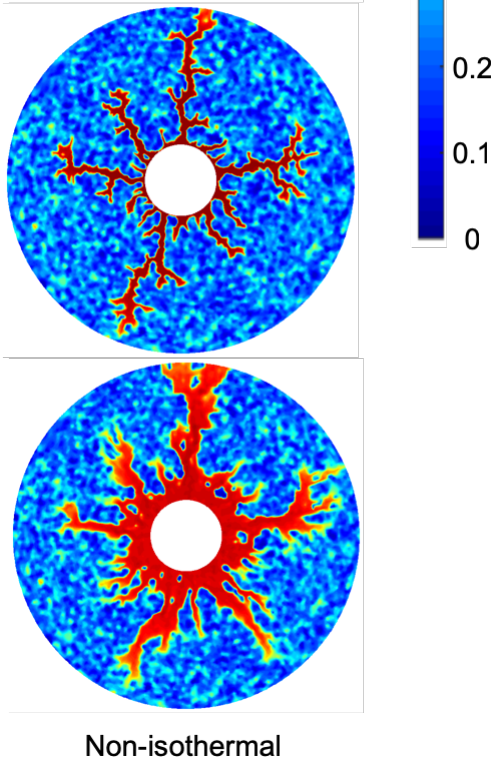

Figure 13: Comparison of dissolution structures for non-isothermal and isothermal cases at different injection rates: (a) $1 / D a=5 \times 10^{-5}$, (b) $1 / D a=3.6 \times 10^{-4}$, (c) $1 / D a=0.008$, (d) $1 / D a=0.06$, (e) $1 / D a=0.5$ 
(a)
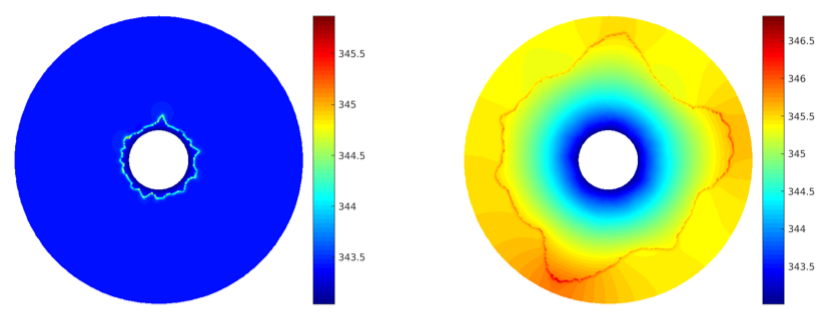

(b)
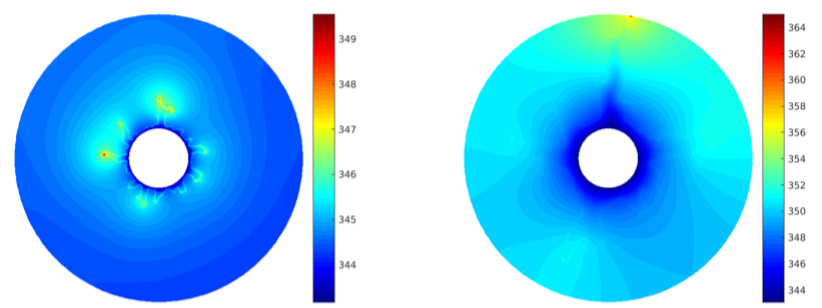

(c)
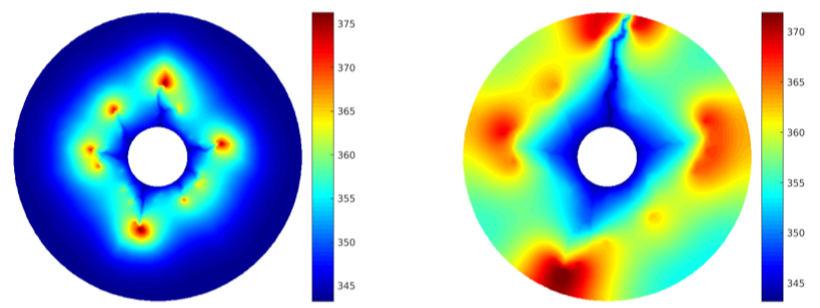

(d)
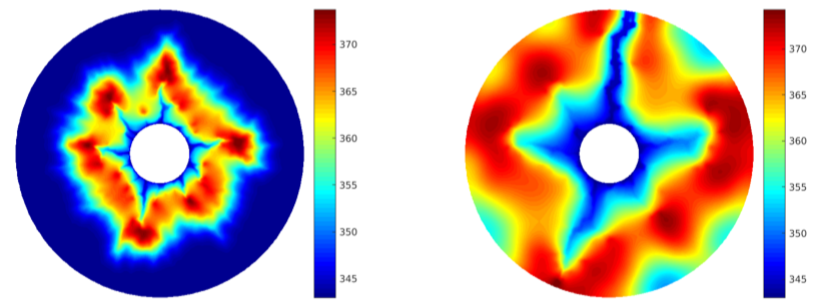

(e)

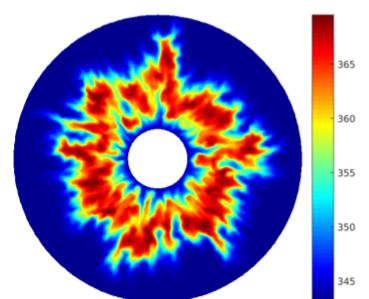

(A)

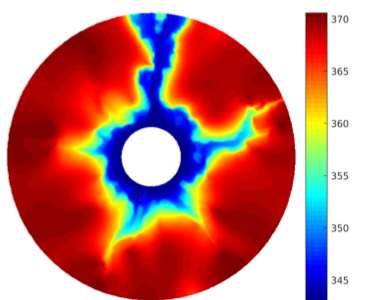

(B)

Figure 14: Temperature distributions at $(\mathrm{A}) t_{D}=0.5$ and (B) breakthrough time for various injection rate: (a) $1 / D a=5 \times 10^{-5}$, (b) $1 / D a=3.6 \times 10^{-4}$, (c) $1 / D a=0.008$, (d) $1 / D a=0.06$, (e) $1 / D a=0.5$ 

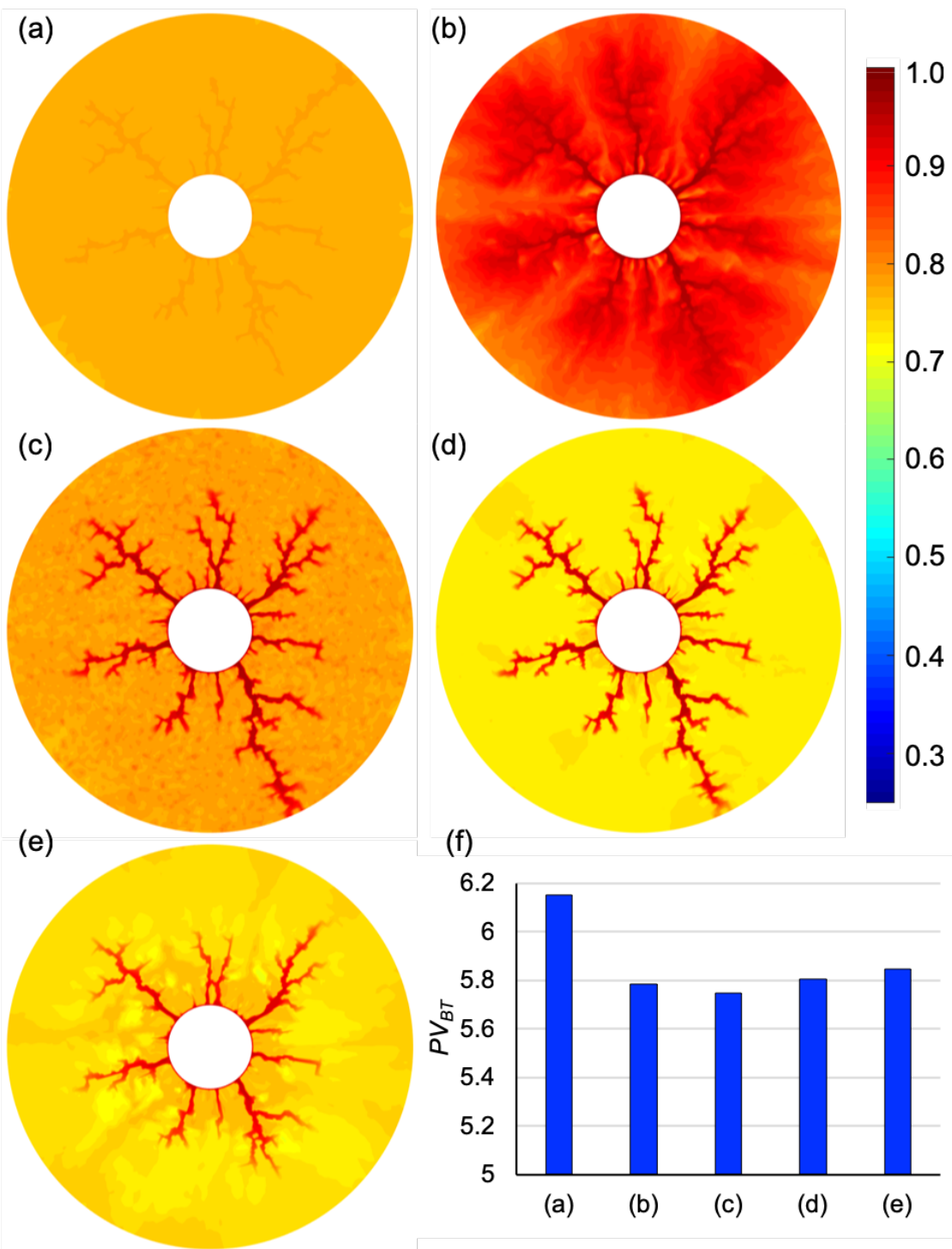

Figure 15: Effect of $a$ value on water saturation at the breakthrough time as well as $P V_{B T}$ : (a) fixed relative permeability curve, (b) $a=10^{-4}$, (c) $a=1$, (d) $a=5$, (e) $a=100$, (f) breakthrough volume. 

under the non-isotherm condition is investigated here.

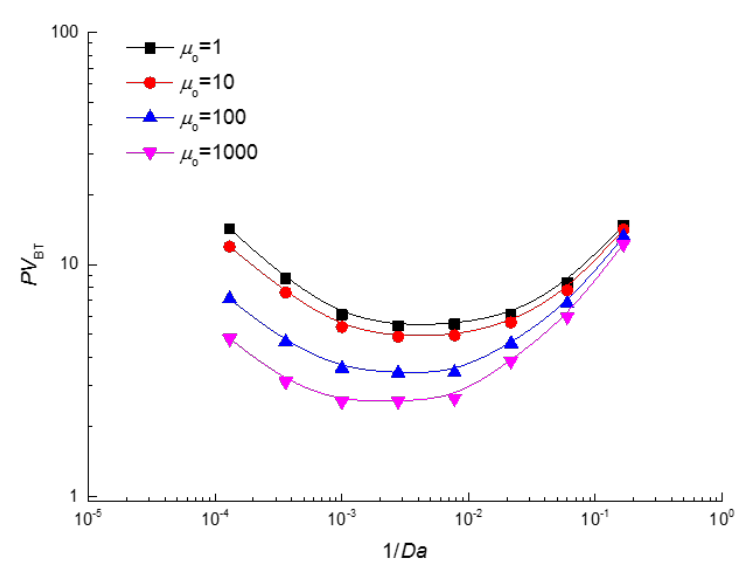

Figure 16: Effect of oil viscosity on breakthrough volume

sweep efficiency and hence most injected acid flows into the dominate wormhole causing the fact that less acid is consumed when breakthrough, Mahmoodi [37] ascribed the decrease in $P V_{B T}$ to a reduction in acid leakage from the dissolved channel to its surrounding zone and the viscous fingering. To clarify, the effect of oil-water viscosity ratio on dissolution dynamic

(1)

The initial temperature of the injected acid and the rock saturated with oil is set to $298 \mathrm{~K}$ and $343 \mathrm{~K}$, respectively. The water viscosity is fixed at $1 \mathrm{mPa} \cdot \mathrm{s}$ while the oil viscosity is varying. Four cases with $\mu_{o}=1 \mathrm{mPa} \cdot \mathrm{s}, \mu_{o}=10 \mathrm{mPa} \cdot \mathrm{s}, \mu_{o}=100 \mathrm{mPa} \cdot \mathrm{s}$, and $\mu_{o}=$ $1000 \mathrm{mPa} \cdot \mathrm{s}$ are simulated. In these simulation cases, the viscosity of water and oil is assumed as temperature independent. This choice allows us to focus on the effect of oilwater viscosity ratio on the dissolution dynamic, without any complications from changes to viscosity ratio itself. Fig. 16 shows the breakthrough curves for cases with various oil viscosity. It can be seen that lower $P V_{B T}$ is obtained for the rock saturated with oil that has high viscosity, which confirms the conclusion obtained by Wei [36] and Mahmoodi [37].

To examine the mechanism behind this phenomenon, the wormhole structures obtained at the optimum injection rate for various oil viscosity are depicted in Fig. 17(A). It can be seen that, except for the dominate wormhole, the length of other wormholes is shorter for the case with higher oil viscosity, and the branchiness of the wormhole decreases as the oil viscosity increases. Because high oil viscosity will result in a poor sweeping efficiency overall, the saturation of the immovable oil increases with oil viscosity increasing, as can be seen in Fig. 17(B), where the water saturation at the breakthrough time is displayed. For example, when oil viscosity is $1 \mathrm{mPa} \cdot \mathrm{s}$, the oil saturation in the core is close to the residual 
(a)
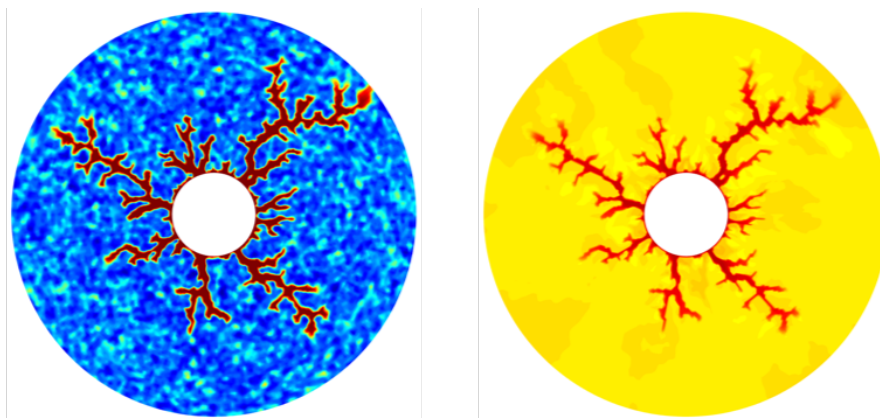

(b)
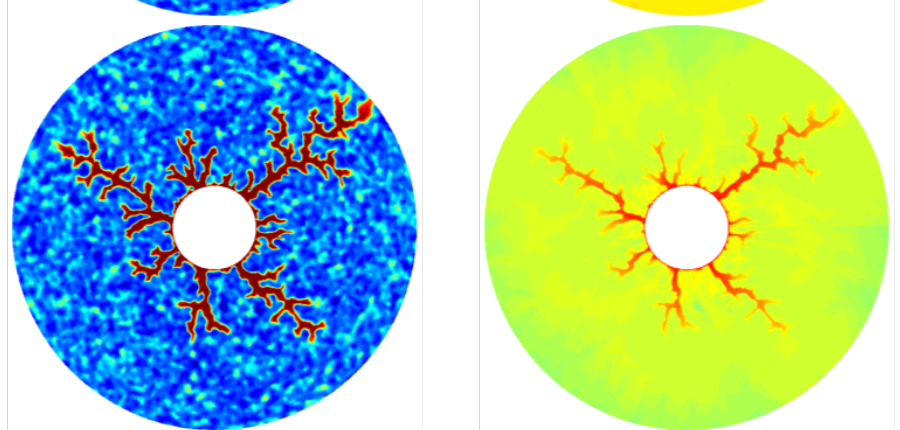

(c)
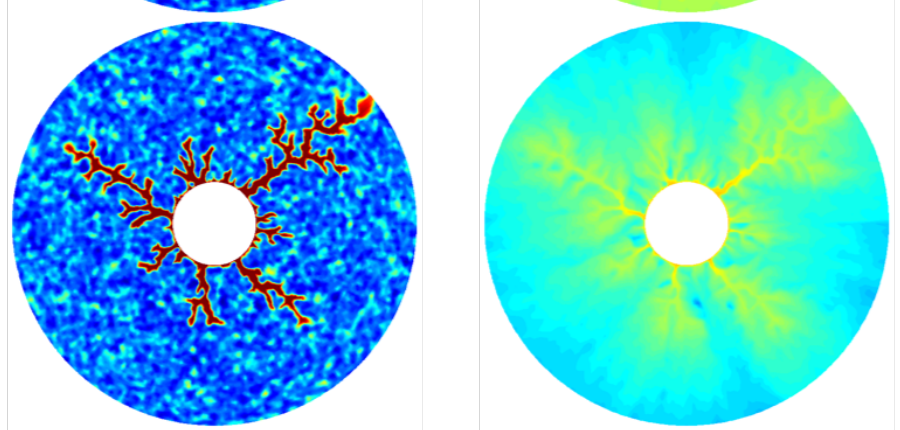

(d)

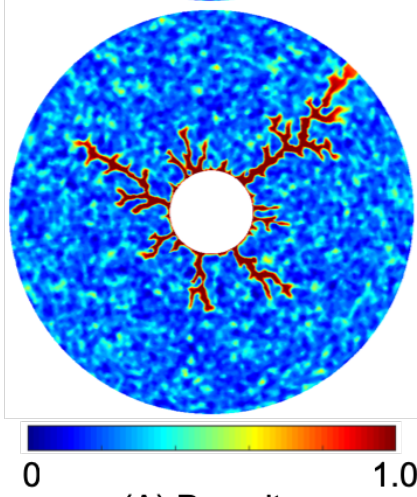

(A) Porosity

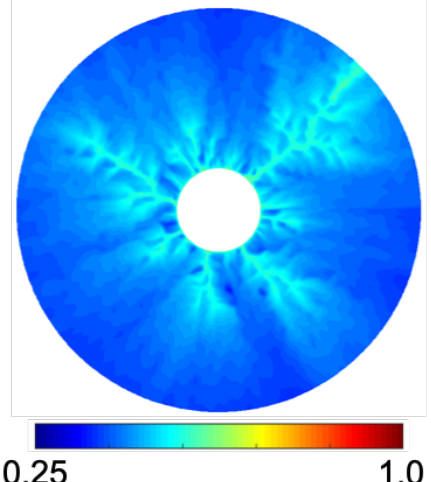

(B) Water saturation

Figure 17: Porisity (A) and acid saturation (B) distributions for various oil viscosity cases at breakthrough time: (a) $\mu_{o}=1 \mathrm{mPa} \cdot \mathrm{s}$, (b) $\mu_{o}=10 \mathrm{mPa} \cdot \mathrm{s}$, (c) $\mu_{o}=100 \mathrm{mPa} \cdot \mathrm{s}$, (d) $\mu_{o}=1000 \mathrm{mPa} \cdot \mathrm{s}$ 
oil saturation after displacement, while when oil viscosity is $1000 \mathrm{mPa} \cdot \mathrm{s}$, the saturation of the oil in the core by the end of the displacement is still high. The high saturation of oil phase reduces the relative permeability of water phase and hence makes it diffcult for injected acid to flow into the surrounding area of wormhole, which leads to the formation of shorter wormholes with lower branchiness, and consequently a lower $P V_{B T}$ because the acid is consumed for dissolving the rock. However, this lower $P V_{B T}$ does not always indicate a high efficiency for acidizing treatment because in this case only small part of formation is stimulated by the acid. Therefore, to get a uniform stimulation, it is necessary to inject a pre-flulsh before injecting the acid if the formation is initially saturated with high viscosity oil.

\section{Conclusions}

The main contribution of this work is the development of a two-phase thermal-hydrologicchemical coupled model that can simulate the dissolution process of carbonate acidizing under non-isotherm condition. Particularly, a new model is developed to describe the change in irreducible water saturation, residual oil saturation, and the maximum relative permeability of oil and water phases with dissolution proceeding. A new method for generation of the initial porosity field is also presented. Unlike the upwind scheme used in previous works for discretizing the convection term, the MINMOD scheme based on the TVD framework is used in this work. The dissolution patterns and breakthrough curves calculated using these two schemes are compared, and it is found that the upwind scheme will introduce stream wise diffusion, which leads to an overestimation of $P V_{B T}$ and an enlargement of the wormhole diameter. Hence, the upwind scheme is not recommended when simulating the reactive flow problem. By analyzing the influence of rock properties, the exothermic heat of reaction, and two-phase flow, on the dissolution process, the following conclusions are obtained:

1. The $P V_{B T}$ predictions are independent of the permeability heterogeneity magnitude at very high and very low injection rates. However, at intermediate injection rate, which corresponds to the wormhole regime, both the $P V_{B T}$ predictions and wormhole branchiness are influenced by the heterogeneity magnitude.

2. The formed wormhole is directional in anisotropic formations. Therefore, self-diverting acids are recommended to make all reservoir zones being treated sufficiently.

3. Temperature is a critical parameter in wormhole propagation and hence should be considered when designing the acidizing stimulation treatment. 
4. The acid volume required for the generation of wormholes in a rock saturated with high viscosity oil is less than that saturated with low viscosity oil. Nevertheless, injecting the acid directly into the formation that is saturated with high viscosity oil is not recommended because in this case only small part of formation is stimulated by the acid.

It should be noted that in this work we mainly focus on the development of the model and the construction of the solving algorithms, so only limited simulation result is presented, and the factors that governed the wormhole formation are analyzed individually. A more detailed analysis of the mutual influence of these factors will be presented in our future work. Regarding the effect of the presence of a non-aqueous phase on wormhole formation, a change in the optimum injection rate has been observed in the experiment studies [37], but not appeared in our simulation results. This may be because there are other proposed mechanisms that are not considered in our model. Therefore, a more detailed model is needed to account for other possible mechanisms such as the natural diverting effects of blocked pore throats, and the effect of reaction produced $\mathrm{CO}_{2}$. Moreover, wormhole formation is more complex than is usually assumed, and the number of the factors affecting wormhole formation is more than that mentioned in this work. One obvious influence factor that is not considered in the current work but has a significant effect on wormhole formation is the stress existed in the actual reservoir [77] . Therefore, a model providing a fundamental understanding of the interplay between the coupled T-H-C process and mechanical compaction also needs to be developed. Some of these further investigations will be pursued in future work.

\section{Acknowledgments}

The authors gratefully acknowledge the support from the National Natural Science Foundation of China (Nos. 51804325, 51874262, 51774317).

\section{References}

[1] M. J. Economides, K. G. Nolte, et al., Reservoir stimulation, Vol. 2, Prentice Hall Englewood Cliffs, NJ, 1989.

[2] C. Fredd, H. Fogler, Alternative stimulation fluids and their impact on carbonate acidizing, SPE Journal 4 (03) (1998) 196-205. doi:doi.org/10.2118/31074-MS.

[3] C. Fredd, H. Fogler, Optimum conditions for wormhole formation in carbonate porous media: Influence of transport and reaction, SPE Journal 4 (03) (1999) 196-205.

[4] C. N. Fredd, H. S. Fogler, Influence of transport and reaction on wormhole formation in porous media, AIChE Journal 44 (9) (1998) 1933-1949. 
[5] G. Daccord, R. Lenormand, O. Lietard, Chemical dissolution of a porous medium by a reactive fluid - i. model for the "wormholing" phenomenon, Chemical Engineering Science 48 (1) (1993) 169-178.

[6] G. Daccord, Chemical dissolution of a porous medium by a reactive fluid, Physical review letters 58 (5) (1987) 479.

[7] P. B. Kelemen, J. Whitehead, E. Aharonov, K. A. Jordahl, Experiments on flow focusing in soluble porous media, with applications to melt extraction from the mantle, Journal of Geophysical Research: Solid Earth 100 (B1) (1995) 475-496.

[8] F. Golfier, C. ZARCONE, B. Bazin, R. Lenormand, D. Lasseux, M. QUINTARD, On the ability of a darcy-scale model to capture wormhole formation during the dissolution of a porous medium, Journal of fluid Mechanics 457 (2002) 213-254.

[9] S. Siddiqui, H. A. Nasr-El-Din, A. A. Khamees, Wormhole initiation and propagation of emulsified acid in carbonate cores using computerized tomography, Journal of Petroleum Science and Engineering 54 (3) (2006) 93-111.

[10] A. Machado, T. Oliveira, F. Cruz, R. Lopes, I. Lima, X-ray microtomography of hydrochloric acid propagation in carbonate rocks, Applied Radiation and Isotopes 96 (2015) 129-134.

[11] A. Machado, R. Lopes, I. Lima, T. Oliveira, F. Bittencourt, 3d wormhole visualization after carbonate matrix acidizing with edta, in: 13th International Congress of the Brazilian Geophysical Society \& EXPOGEF, Rio de Janeiro, Brazil, 26-29 August 2013, Society of Exploration Geophysicists and Brazilian Geophysical Society, 2013, pp. 1159-1160.

[12] T. Frick, B. Mostofizadeh, M. Economides, Analysis of radial core experiments for hydrochloric acid interaction with limestones, in: SPE Formation Damage Control Symposium, Society of Petroleum Engineers, 1994, pp. 577-592.

[13] K. Dong, X. Jin, D. Zhu, A. Hill, The effect of core dimensions on the optimal acid flux in carbonate acidizing, in: SPE International Symposium and Exhibition on Formation Damage Control, Society of Petroleum Engineers, 2014, pp. 1-10.

[14] C. Karale, A. Beuterbaugh, M. Pinto, G. Hipparge, A. Prakash, Hp/ht carbonate acidizing - recent discoveries and contradictions in wormhole phenomenon, in: Offshore Technology Conference Asia, Offshore Technology Conference, 2016, pp. 1-23.

[15] H. Cheng, D. Zhu, A. Hill, The effect of evolved co 2 on wormhole propagation in carbonate acidizing, in: SPE International Conference and Exhibition on Formation Damage Control, Society of Petroleum Engineers, 2017, pp. 1-12.

[16] B. Bazin, From matrix acidizing to acid fracturing: A laboratory evaluation of acid/rock interactions, SPE Production \& Facilities 16 (01) (2001) 22-29.

[17] M. E. Ziauddin, E. Bize, et al., The effect of pore scale heterogeneities on carbonate stimulation treatments, in: SPE Middle East Oil and Gas Show and Conference, Society of Petroleum Engineers, 2007, pp. 1-14.

[18] Y. Wang, A. Hill, R. Schechter, et al., The optimum injection rate for matrix acidizing of carbonate formations, in: SPE Annual Technical Conference and Exhibition, Society of Petroleum Engineers, 1993, pp. 675-687.

[19] M. A. Al-Obied, I. AlYami, A. Shebatalhamd, D. A. Al-Shehri, et al., Wormhole propagation in tar during matrix acidizing of carbonate formations, in: SPE International Symposium and Exhibition on 
Formation Damage Control, Society of Petroleum Engineers, 2012, pp. 1-15.

[20] R. Kumar, J. He, H. Nasr-El-Din, et al., Effect of oil saturation on acid propagation during matrix acidization of carbonate rocks, in: SPE Latin America and Caribbean Petroleum Engineering Conference, Society of Petroleum Engineers, 2014, pp. 1-22.

[21] S. Shukla, D. Zhu, A. Hill, et al., The effect of phase saturation conditions on wormhole propagation in carbonate acidizing, Spe Journal 11 (03) (2006) 273-281.

[22] M. K. Panga, M. Ziauddin, V. Balakotaiah, Two-scale continuum model for simulation of wormholes in carbonate acidization, AIChE Journal 51 (12) (2005) 3231-3248.

[23] N. Kalia, V. Balakotaiah, Modeling and analysis of wormhole formation in reactive dissolution of carbonate rocks, Chemical Engineering Science 62 (4) (2007) 919-928. doi:http://dx.doi.org/10. $1016 / j$.ces. 2006.10 .021 .

URL http://www.sciencedirect.com/science/article/pii/S0009250906006713

[24] C. E. Cohen, D. Ding, M. Quintard, B. Bazin, From pore scale to wellbore scale: Impact of geometry on wormhole growth in carbonate acidization, Chemical Engineering Science 63 (12) (2008) 3088-3099.

[25] P. Liu, J. Yao, G. D. Couples, J. Ma, O. Iliev, 3-d modelling and experimental comparison of reactive flow in carbonates under radial flow conditions, Scientific Reports 7 (1) (2017) 17711. doi:10.1038/ s41598-017-18095-2.

URL https://doi.org/10.1038/s41598-017-18095-2

[26] M. Liu, S. Zhang, J. Mou, Effect of normally distributed porosities on dissolution pattern in carbonate acidizing, Journal of Petroleum Science and Engineering 94 (2012) 28-39.

[27] R. Ratnakar, N. Kalia, V. Balakotaiah, Carbonate matrix acidizing with gelled acids: An experimentbased modeling study, in: SPE International Production and Operations Conference \& Exhibition, Society of Petroleum Engineers, 2012, pp. 1-16.

[28] R. R. Ratnakar, N. Kalia, V. Balakotaiah, Modeling, analysis and simulation of wormhole formation in carbonate rocks with in situ cross-linked acids, Chemical Engineering Science 90 (2013) 179-199.

[29] P. Maheshwari, J. Maxey, V. Balakotaiah, Reactive-dissolution modeling and experimental comparison of wormhole formation in carbonates with gelled and emulsified acids, SPE J 5 (2016) 103-119.

[30] P. Liu, J. Yao, G. D. Couples, Z. Huang, H. Sun, J. Ma, Numerical modelling and analysis of reactive flow and wormhole formation in fractured carbonate rocks, Chemical Engineering Science 172 (2017) 143-157. doi:10.1016/j.ces.2017.06.027.

[31] P. Liu, J. Yao, G. D. Couples, J. Ma, Z. Huang, H. Sun, Modelling and simulation of wormhole formation during acidization of fractured carbonate rocks, Journal of Petroleum Science and Engineering 154 (2017) 284-301. doi:10.1016/j.ces.2017.06.027.

[32] N. Kalia, G. Glasbergen, Wormhole formation in carbonates under varying temperature conditions, in: European Formation Damage Conference, Society of Petroleum Engineers, 2009, pp. 1-19.

[33] N. Kalia, G. Glasbergen, Fluid temperature as a design parameter in carbonate matrix acidizing, in: SPE Production and Operations Conference and Exhibition, Society of Petroleum Engineers, 2009, pp. $1-21$.

[34] Y. Li, Y. Liao, J. Zhao, Y. Peng, X. Pu, Simulation and analysis of wormhole formation in carbonate rocks considering heat transmission process, Journal of Natural Gas Science and Engineering 42 (2017) 120-132. 
[35] Y. Li, Q. Deng, J. Zhao, Y. Liao, Y. Jiang, Simulation and analysis of matrix stimulation by diverting acid system considering temperature field, Journal of Petroleum Science and Engineering 170 (2018) 932-944.

[36] W. Wei, A. Varavei, K. Sepehrnoori, et al., Modeling and analysis on the effect of two-phase flow on wormhole propagation in carbonate acidizing, SPE Journal 22 (06) (2017) 2067-2083.

[37] A. Mahmoodi, A. Javadi, B. S. Sola, Porous media acidizing simulation: New two-phase two-scale continuum modeling approach, Journal of Petroleum Science and Engineering 166 (2018) 679-692.

[38] M. Babaei, M. Sedighi, Impact of phase saturation on wormhole formation in rock matrix acidizing, Chemical Engineering Science 177 (2018) 39-52.

[39] Y. Wu, A. Salama, S. Sun, Parallel simulation of wormhole propagation with the darcy-brinkmanforchheimer framework, Computers and Geotechnics 69 (2015) 564-577.

[40] T. Yuan, Y. Ning, G. Qin, Numerical modeling and simulation of coupled processes of mineral dissolution and fluid flow in fractured carbonate formations, Transport in Porous Media 114 (3) (2016) $747-775$.

[41] P. Liu, G. D. Couples, J. Yao, Z. Huang, W. Song, J. Ma, A general method for simulating reactive dissolution in carbonate rocks with arbitrary geometry, Computational Geosciences (2018) 1-15.

[42] A. Bastami, P. Pourafshary, Development of a new model for carbonate matrix acidizing to consider the effects of spent acid, Journal of Energy Resources Technology 138 (5) (2016) 052905.

[43] A. Safari, M. M. Dowlatabad, A. Hassani, F. Rashidi, Numerical simulation and x-ray imaging validation of wormhole propagation during acid core-flood experiments in a carbonate gas reservoir, Journal of Natural Gas Science and Engineering 30 (2016) 539-547.

[44] P. Liu, H. Xue, L. Zhao, X. Zhao, M. Cui, Simulation of 3d multi-scale wormhole propagation in carbonates considering correlation spatial distribution of petrophysical properties, Journal of Natural Gas Science and Engineering 32 (2016) 81-94.

[45] Y. Jun, L. Piyang, H. Zhaoqin, W. Yueying, Y. Xia, Z. Qingdong, Status and progress of reactive flow simulations for carbonate reservoirs, Earth Science 42 (8) (2017) 1263-1272.

[46] J. YAO, P. LIU, Z. HUANG, Y. WANG, X. YAN, Analysis of influencing factors on the optimum stimulation conditions of the acidizing treatment in carbonate reservoirs, Scientia Sinica Technologica 47 (7) (2017) 692-707.

[47] P. Kelemen, G. Hirth, N. Shimizu, M. Spiegelman, H. Dick, A review of melt migration processes in the adiabatically upwelling mantle beneath oceanic spreading ridges, Philosophical Transactions of the Royal Society of London. Series A: Mathematical, Physical and Engineering Sciences 355 (1723) (1997) 283-318.

[48] M. Daines, D. Kohlstedt, The transition from porous to channelized flow due to melt/rock reaction during melt migration, Geophysical Research Letters 21 (2) (1994) 145-148.

[49] C. Steefel, C. Appelo, B. Arora, D. Jacques, T. Kalbacher, O. Kolditz, V. Lagneau, P. Lichtner, K. U. Mayer, J. Meeussen, et al., Reactive transport codes for subsurface environmental simulation, Computational Geosciences 19 (3) (2015) 445-478.

[50] L. Chen, Q. Kang, B. Carey, W.-Q. Tao, Pore-scale study of diffusion-reaction processes involving dissolution and precipitation using the lattice boltzmann method, International Journal of Heat and Mass Transfer 75 (2014) 483-496. 
[51] T. Xu, E. Sonnenthal, N. Spycher, K. Pruess, Toughreact - a simulation program for non-isothermal multiphase reactive geochemical transport in variably saturated geologic media: applications to geothermal injectivity and co2 geological sequestration, Computers \& Geosciences 32 (2) (2006) 145-165.

[52] Q. Kang, I. N. Tsimpanogiannis, D. Zhang, P. C. Lichtner, Numerical modeling of pore-scale phenomena during co2 sequestration in oceanic sediments, Fuel processing technology 86 (14-15) (2005) 1647-1665.

[53] R. Zhang, Y. Xiong, P. H. Winterfeld, X. Yin, Y.-S. Wu, A novel computational framework for thermalhydrological-mechanical-chemical processes of co2 geological sequestration into a layered saline aquifer and a naturally fractured enhanced geothermal system, Greenhouse Gases: Science and Technology 6 (3) (2016) 370-400.

[54] J. Yow, J. Hunt, Coupled processes in rock mass performance with emphasis on nuclear waste isolation, International journal of rock mechanics and mining sciences 39 (2) (2002) 143-150.

[55] G.-T. Yeh, Y. Fang, F. Zhang, J. Sun, Y. Li, M.-H. Li, M. D. Siegel, Numerical modeling of coupled fluid flow and thermal and reactive biogeochemical transport in porous and fractured media, Computational Geosciences 14 (1) (2010) 149-170.

[56] A. Karrech, Non-equilibrium thermodynamics for fully coupled thermal hydraulic mechanical chemical processes, Journal of the Mechanics and Physics of Solids 61 (3) (2013) 819-837.

[57] O. Kolditz, S. Bauer, L. Bilke, N. Böttcher, J.-O. Delfs, T. Fischer, U. J. Görke, T. Kalbacher, G. Kosakowski, C. McDermott, et al., Opengeosys: an open-source initiative for numerical simulation of thermo-hydro-mechanical/chemical (thm/c) processes in porous media, Environmental Earth Sciences 67 (2) (2012) 589-599.

[58] C. McDermott, A. Bond, A. F. Harris, N. Chittenden, K. Thatcher, Application of hybrid numerical and analytical solutions for the simulation of coupled thermal, hydraulic, mechanical and chemical processes during fluid flow through a fractured rock, Environmental Earth Sciences 74 (12) (2015) $7837-7854$

[59] J. Taron, D. Elsworth, K.-B. Min, Numerical simulation of thermal-hydrologic-mechanical-chemical processes in deformable, fractured porous media, International Journal of Rock Mechanics and Mining Sciences 46 (5) (2009) 842-854.

[60] H. R. Thomas, M. Sedighi, P. J. Vardon, Diffusive reactive transport of multicomponent chemicals under coupled thermal, hydraulic, chemical and mechanical conditions, Geotechnical and Geological Engineering 30 (4) (2012) 841-857.

[61] J. Chen, S. Sun, Z. Chen, Coupling two-phase fluid flow with two-phase darcy flow in anisotropic porous media, Advances in Mechanical Engineering 6 (2014) 871021.

[62] T. Al-Shemmeri, Engineering fluid mechanics, Bookboon, 2012.

[63] Z. Huang, X. Yan, J. Yao, A two-phase flow simulation of discrete-fractured media using mimetic finite difference method, Communications in Computational Physics 16 (3) (2014) 799-816.

[64] V. K. Upadhyay, P. Szymczak, A. J. Ladd, Initial conditions or emergence: What determines dissolution patterns in rough fractures?, Journal of Geophysical Research: Solid Earth 120 (9) (2015) 6102-6121.

[65] N. Kalia, V. Balakotaiah, Effect of medium heterogeneities on reactive dissolution of carbonates, Chemical Engineering Science 64 (2) (2009) 376-390.

[66] C. Wilke, P. Chang, Correlation of diffusion coefficients in dilute solutions, AIChE Journal 1 (2) (1955) 264-270. 
[67] P. Maheshwari, R. R. Ratnakar, N. Kalia, V. Balakotaiah, 3-d simulation and analysis of reactive dissolution and wormhole formation in carbonate rocks, Chemical Engineering Science 90 (0) (2013) 258-274. doi:http://dx.doi.org/10.1016/j.ces.2012.12.032.

URL http://www.sciencedirect.com/science/article/pii/S0009250912007233

[68] D. Tiab, E. C. Donaldson, Petrophysics: theory and practice of measuring reservoir rock and fluid transport properties, Gulf professional publishing, 2015.

[69] J. Jensen, L. W. Lake, P. W. Corbett, D. Goggin, Statistics for petroleum engineers and geoscientists, Vol. 2, Gulf Professional Publishing, 2000.

[70] A. G. Journel, Geostatistics for conditional simulation of ore bodies, Economic Geology 69 (5) (1974) 673-687.

[71] D. S. Oliver, Moving averages for gaussian simulation in two and three dimensions, Mathematical Geology 27 (8) (1995) 939-960.

[72] J. Li, B. Yu, Y. Wang, Y. Tang, H. Wang, Study on computational efficiency of composite schemes for convection-diffusion equations using single-grid and multigrid methods, Journal of Thermal Science and Technology 10 (1) (2015) JTST0009-JTST0009.

[73] G. Zhu, H. Chen, J. Yao, S. Sun, Efficient energy-stable schemes for the hydrodynamics coupled phasefield model, Applied Mathematical Modelling 70 (2019) 82-108.

[74] D. McDuff, C. E. Shuchart, S. Jackson, D. Postl, J. S. Brown, Understanding wormholes in carbonates: Unprecedented experimental scale and 3-d visualization, in: SPE Annual Technical Conference and Exhibition, Society of Petroleum Engineers, 2010, pp. 1-9.

[75] T. J. L. De Oliveira, A. R. De Melo, J. A. A. Oliveira, A. Z. Pereira, et al., Numerical simulation of the acidizing process and pvbt extraction methodology including porosity/permeability and mineralogy heterogeneity, in: SPE International Symposium and Exhibition on Formation Damage Control, Society of Petroleum Engineers, 2012, pp. 1-9.

[76] P. Maheshwari, V. Balakotaiah, Comparison of carbonate hcl acidizing experiments with 3d simulations, SPE J 28 (04) (2013) 402-413.

[77] X. Yan, Z. Huang, J. Yao, Z. Zhang, P. Liu, Y. Li, D. Fan, Numerical simulation of hydro-mechanical coupling in fractured vuggy porous media using the equivalent continuum model and embedded discrete fracture model, Advances in Water Resources 126 (2019) 137-154. 